\title{
X-ray absorption spectroscopy of Ru-doped relaxor ferroelectrics with a perovskite-type structure
}

\author{
T. Vitova,,${ }^{1, *}$ S. Mangold, ${ }^{2}$ C. Paulmann, ${ }^{3}$ M. Gospodinov,${ }^{4}$ V. Marinova, ${ }^{5}$ and B. Mihailova ${ }^{3, \dagger}$ \\ ${ }^{1}$ Institut für Nukleare Entsorgung, Karlsruher Institut für Technologie, Hermann-von-Helmholtz-Platz 1, \\ D-76344 Eggenstein-Leopoldshafen, Germany \\ ${ }^{2}$ ANKA Synchrotronstrahlungsquelle, Karlsruher Institut für Technologie, Hermann-von-Helmholtz-Platz 1, \\ D-76344 Eggenstein-Leopoldshafen, Germany \\ ${ }^{3}$ Fachbereich Geowissenschaften, Universität Hamburg, Grindelallee 48, 20146 Hamburg, Germany \\ ${ }^{4}$ Institute of Solid State Physics, Bulgarian Academy of Sciences, Blvd. Tzarigradsko Chaussee 72, 1784 Sofia, Bulgaria \\ ${ }^{5}$ Institute of Optical Materials and Technologies, Bulgarian Academy of Sciences, Acad. G. Bontchev Str. 109, 1113 Sofia, Bulgaria
}

(Received 17 July 2013; revised manuscript received 16 April 2014; published 29 April 2014)

\begin{abstract}
$\mathrm{X}$-ray absorption near-edge structure and extended $\mathrm{x}$-ray absorption fine structure spectroscopy at the $\mathrm{Ru}$ $K$ edge of Ru-doped $\mathrm{PbSc}_{0.5} \mathrm{Ta}_{0.5} \mathrm{O}_{3}$ (PST-Ru), $\mathrm{PbSc}_{0.5} \mathrm{Nb}_{0.5} \mathrm{O}_{3}$ (PSN-Ru), and $0.9 \mathrm{PbZn}_{1 / 3} \mathrm{Nb}_{2 / 3} \mathrm{O}_{3}-0.1 \mathrm{PbTiO}_{3}$ (PZN-0.1PT-Ru) as well as at the Ta $L_{3}$ edge of PST-Ru and the Nb $K$ edge of PSN-Ru was applied to study the short- and intermediate-range atomic arrangements in perovskite-type $\left(\mathrm{ABO}_{3}\right)$ relaxor ferroelectrics. The compounds were also analyzed by complementary Raman scattering, visible/near-visible absorption spectroscopy, and synchrotron x-ray single-crystal diffraction. The results show that $\mathrm{Ru}$ is octahedrally coordinated in all three relaxor host matrices but the average oxidation state of Ru in PST-Ru and PSN-Ru is $\sim 4.4$, whereas it is $\sim 3.8$ in PZN-0.1PT-Ru. In $\mathrm{PbSc}_{0.5} B^{\prime \prime}{ }_{0.5} \mathrm{O}_{3}\left(B^{\prime \prime}=\mathrm{Ta}\right.$, Nb) Ru substitutes for the $B^{\prime \prime}$ cations in the form of isolated point defects, while in PZN-0.1PT-Ru Ru replaces adjacent $A$ and $B$ sites, forming a chainlike structural species of face-sharing elongated octahedra. Chemical $1: 1 B$-site order as well as dynamic $B \mathrm{O}_{6}$ tilting is observed around both the Ru dopant and the major $B^{\prime \prime}$ cation in PST-Ru and PSN-Ru regardless of the fact that according to X-ray diffraction at ambient conditions, the average structure is cubic with weak or no long-range chemical order. $\mathrm{Pb}$ cations are off-center displaced from the prototypic cubic $A$ site for all three compounds and in $\mathrm{Ru}$-doped $\mathrm{PbSc}_{0.5} B^{\prime \prime}{ }_{0.5} \mathrm{O}_{3}$ the $B \mathrm{O}_{6}$ tilt angle correlates with the degree of coherent $B$ - $\mathrm{Pb}$ distances.
\end{abstract}

DOI: 10.1103/PhysRevB.89.144112

PACS number(s): 77.80.Jk, 61.05.cj, 61.72.J-, 78.30.-j

\section{INTRODUCTION}

The search for advanced materials with high response functions over the past two decades has spotted complex perovskite-type relaxor-ferroelectric materials of the general formula $A B \mathrm{O}_{3}$, with $\mathrm{Pb}^{2+}$ on the $A$ site and various transition elements on the $B$ site [1-4]. In contrast to conventional ferroelectrics, relaxor ferroelectrics exhibit a broad and frequency-dependent maximum of the dielectric permittivity as a function of temperature due to the existence of the so-called polar nanoregions: spatial regions with coherent local polar distortions on the mesoscopic scale, which near and above the temperature of the dielectric permittivity maximum $T_{m}$ flip between different crystallographically and energetically equivalent orientation states [1,2]. The dynamic polar nanoregions are surrounded by a paraelectric matrix comprised of uncoupled dynamic local ferroic distortions [5] which on average produce no polarity and thus the relaxor state can be also considered as dynamic "nanodomains" separated by domain walls with a mean thickness nearly the same as the mean domain size [6]. These dynamic polar nanoregions are believed to be responsible for the remarkable relaxor properties such as very high dielectric permittivity and very strong electroelastic, piezoelectric, pyroelectric, photoelastic, and electro-optic effects, which makes relaxors and related systems important technological materials with applications in actuators, capacitors, medical imaging devices,

\footnotetext{
*Corresponding author: tonya.vitova@kit.edu

†Corresponding author: boriana.mihailova@uni-hamburg.de
}

and memories $[3,4,7,8]$. The mean size and abundance of polar nanoregions can be influenced by chemically induced local elastic and/or electric fields, which highlights the key role of doping in order to tune and enhance the desired macroproperties of relaxor ferroelectrics.

Ruthenium is a mixed-valence photochromic element and its incorporation into the structure of perovskite-type ferroelectrics leads to higher photosensitivity and stronger photorefractive effect close to the red and near-infrared spectral range, which is of significant interest for optical memories [9-11]. In addition, Ru-containing perovskites exhibit nontrivial magnetic properties [12-14], which might be related to the multivalent state of Ru. Recently, piezoresponse force microscopy and hysteresis loop measurements of $0.9 \mathrm{PbZn}_{1 / 3} \mathrm{Nb}_{2 / 3} \mathrm{O}_{3}-0.1 \mathrm{PbTiO}_{3}(\mathrm{PZN}-0.1 \mathrm{PT})$, which is a relaxor-ferroelectric solid solution with a giant piezoelectric effect [8], revealed that the addition of $\mathrm{Ru}$ decreases the ferroelectric domain size, reduces the polar fraction distributed in the pseudocubic matrix, and leads to significant ferroelectric hardening due to the immobilization of domain walls [15]. Hence, the incorporation of Ru into relaxor-ferroelectric host matrices has the potential to induce new properties and thus to improve the multifunctionality of the material, which in turn is a strong motivation for fundamental structural studies of Ru-doped relaxor ferroelectrics.

X-ray absorption spectroscopy (XAS) - including the two varieties, $\mathrm{x}$-ray absorption near-edge structure (XANES) and extended $\mathrm{x}$-ray absorption fine structure (EXAFS) - is sensitive to the average oxidation state and the atomic arrangements in the vicinity of a certain chemical element. XAS has been successfully applied to analyze the site occupancy and 
coordination spheres of $\mathrm{Cu}$ and Fe embedded in ferroelectric $\mathrm{LiNbO}_{3}[16,17]$. Furthermore, due to its short length- and time-scale sensitivity XAS can give a deeper insight into the structure of the relaxor host, because the flipping dynamics as well as the small correlation length of coherent polar distortions hinders the application of conventional diffraction methods. For example, Ti $K$-edge XANES studies of $\mathrm{Pb}_{1-x} \mathrm{La}_{x} \mathrm{Zr}_{0.4} \mathrm{Ti}_{0.6} \mathrm{O}_{3}$ demonstrated that the $\mathrm{Ti}$ atoms are displaced from the octahedral centers even for compositions which according to x-ray diffraction (XRD) exhibit cubic $P m \overline{3} m$ symmetry [18]. XANES and EXAFS analyses of $(1-x) \mathrm{PbSc}_{0.5} \mathrm{Ta}_{0.5} \mathrm{O}_{3}-x \mathrm{PbTiO}_{3}$ revealed two unexpected structural features [19]: (i) With increasing $x$ the predominant direction of local off-centered displacements of the Ti atoms gradually change from $\langle 111\rangle_{\text {cubic }}$ to $\langle 100\rangle_{\text {cubic }}$, whereas the symmetry of the global structure abruptly changes from rhombohedral to tetragonal at $x=0.45$; and (ii) $\mathrm{B}^{3+}-\mathrm{O}-\mathrm{B}^{5+}$ atomic bridges dominate over $B^{3+}-\mathrm{O}-B^{3+}$ and $B^{5+}-\mathrm{O}-B^{5+}$, which indicates a high degree of local NaCl-type chemical order on the $B$ site, although the compounds are chemically disordered according to XRD. Comprehensive EXAFS studies performed by Chen et al [20,21] on a number of perovskitetype relaxors with $\mathrm{Pb}^{2}$ on the $A$ site and two types of cation on the $B$ site led to the conclusion that $\mathrm{Pb}^{2+}$ cations exhibit significant off-centered displacements along $\langle 111\rangle_{\text {cubic }}$ and local antipolar order, suggesting that the relaxor state may originate from the competition between antiferroelectric (AFE) and ferroelectric (FE) interactions. Furthermore, XANES studies of $\mathrm{PbMg}_{1 / 3} \mathrm{Nb}_{2 / 3} \mathrm{O}_{3}$ and PZN-0.12PT showed that the local polarization of the ferroelectrically active $B$-site cation $\mathrm{Nb}^{5+}$ is small and plays only a secondary role as compared to the local polarization of $A$-positioned $\mathrm{Pb}^{2+}$, emphasizing the potential of appropriate doping to tailor the relaxor properties by inducing a mixture of local AFE and FE ordering [22]. These XAS results support the recently proposed idea based on complementary Raman scattering and diffraction studies at nonambient conditions $[5,23,24]$ that the polar nanoregions in relaxors are ferrielectric rather than ferroelectric in nature. Acoustic emission [25,26] and Raman spectroscopic studies under an external electric field [27,28] also revealed a coexistence of FE and AFE coupling within polar nanoregions, implying a resultant ferrielectric order on the mesoscopic scale.

Thus, the aim of this study is twofold: to clarify the way of incorporation of $\mathrm{Ru}$ into ferroelectric host matrices of type $\mathrm{Pb} B^{3+}{ }_{0.5} B^{5+}{ }_{0.5} \mathrm{O}_{3}$ and $\mathrm{Pb} B^{2+}{ }_{1 / 3} B^{5+}{ }_{2 / 3} \mathrm{O}_{3}-\mathrm{PbB}^{4+} \mathrm{O}_{3}$, namely, the oxidation state, local coordination, and possible dopant clustering, as well as to analyze the predominant atomic linkages in the surrounding host matrix by XAS of $\mathrm{Ru}$-doped $\mathrm{PbSc}_{0.5} \mathrm{Ta}_{0.5} \mathrm{O}_{3}$ (PST), $\mathrm{PbSc}_{0.5} \mathrm{Nb}_{0.5} \mathrm{O}_{3}$ (PSN), and PZN-0.1PT. The relaxor compounds were also analyzed by complementary Raman scattering with different excitation wavelengths, optical absorption spectroscopy, and singecrystal x-ray diffraction with synchrotron radiation.

\section{EXPERIMENTAL DETAILS}

\section{A. Sample synthesis and characterization}

Cube-shaped single crystals with an average size $3 \times 3 \times$ $3 \mathrm{~mm}^{3}$ were synthesized by the high-temperature solution growth method using a flux solution with a composition of $\mathrm{PbO}: \mathrm{PbF}_{2}: B_{2} \mathrm{O}_{3}=0.75: 0.24: 0.1$ for PST-Ru and PSN-Ru and $\mathrm{Pb}_{3} \mathrm{O}_{4}: B_{2} \mathrm{O}_{3}=0.75: 0.25$ for PZN-0.1PT-Ru. Ruthenium was added to a powder of the corresponding relaxor compound in the form of $\mathrm{RuO}_{2}$. The ratio between the flux and the raw solid material was 7:1. After initial annealing for $48 \mathrm{~h}$, the synthesis mixture was cooled from $1200{ }^{\circ} \mathrm{C}$ to $920^{\circ} \mathrm{C}$ at a rate of $0.5^{\circ} \mathrm{C} / \mathrm{h}$ for PST-Ru and PSN-Ru and at a rate of $0.3^{\circ} \mathrm{C} / \mathrm{h}$ for PZN-0.1PT-Ru.

The chemical compositions of the grown crystals were determined by electron microprobe analysis (Cameca Microbeam SX100) averaging more than 50-100 points from each specimen. The calculated stoichiometries are given in Table I.

Powder XRD data were collected with a Philips X'Pert diffractometer (Bragg-Brenato geometry), using a $\mathrm{Cu} K \alpha$ radiation. The XRD analysis of PST-Ru and PSN-Ru revealed a cubic structure at ambient conditions for both compounds (see Table I). Due to the presence of partial long-range 1:1 $B$-site chemical order, PST-Ru has a face-centered-cubic symmetry, in contrast to PSN-Ru, which is entirely chemically disordered according to powder XRD. Pure PZN-0.1PT is at the morphotropic phase boundary (MPB) of the $(1-x) \mathrm{PZN}$ $x \mathrm{PT}$ solid solution and hence its structural state is very complex. The MPB structure of $(1-x) \mathrm{PZN}-x \mathrm{PT}$ has been extensively studied by several groups using high-precision neutron diffraction and synchrotron XRD and it has been shown that ferroelectric monoclinic domains are predominant at room temperature and ambient pressure [30,31]. Previous temperature-dependent XRD and Raman scattering studies of PZN-0.1PT and PZN-0.1PT-Ru from the same synthesis batch as the sample studied here revealed that at room temperature the structure of PZN-0.1PT-Ru resembles that of PZN-0.1PT [23]. According to high-precision singlecrystal XRD data on PZN-01.PT at ambient conditions [32], refinements of the unit-cell parameters in a monoclinic metric give $a=4.0306(3) \AA, b=4.0939(3), c=4.03020(3) \AA$, $\alpha=89.96^{\circ}\left(1^{\circ}\right), \beta=\gamma=90.00^{\circ}$, and a unit-cell volume $V=66.533(9) \AA^{3}$. Thus as initial input crystallographic data for calculating the scattering paths in the EXAFS analysis of PZN-0.1PT-Ru we used a pseudocubic unit-cell parameter $a=\sqrt[3]{V}$ calculated from the unit-cell volume $V$ of PZN-0.1PT determined by high-precision XRD analysis [32].

The samples were also analyzed by single-crystal XRD with a synchrotron radiation at the F1 beamline of HASYLAB/DESY using a MarCCD 165 detector and a radiation wavelength of $\lambda=0.4000 \AA$ as well as by Raman spectroscopy (Horiba T64000 triple grating spectrometer with an Olympus microscope) with an excitation wavelength $\lambda=514.5 \mathrm{~nm}$ and $\lambda=325 \mathrm{~nm}$. PST-Ru was also probed by Fourier-transform (FT) Raman spectroscopy with $\lambda=$ 1064 nm (Bruker IFS 66 FT-IR spectrometer with an FRA 106 FT-Raman module). Additionally, PST-Ru and PZN-0.1PT-Ru were studied by visible/near-visible spectroscopy (Jasco 550 UV-VIS spectrophotometer and Perkin Elmer Lambda 900 UV/VIS/NIR spectrometer). All Raman and optical spectroscopic experiments were performed at room temperature, using polished plates parallel to one of the cubic $\{100\}$ planes. 
TABLE I. Sample nomenclature and characteristics.

\begin{tabular}{lllc}
\hline \hline & & \multicolumn{1}{c}{$\begin{array}{c}\text { Symmetry and unit-cell } \\
\text { parameters at ambient conditions }\end{array}$} & $\begin{array}{c}\text { Degree of long-range chemical } \\
B \text {-site order } / \text { mean size of } \\
\text { chemically ordered domains }\end{array}$ \\
\hline PST-Ru & $\mathrm{PbSc}_{0.480} \mathrm{Ta}_{0.510} \mathrm{Ru}_{0.010} \mathrm{O}_{3}$ & $F m \overline{3} m, 2 a=8.135(3) \AA$ & $0.12 / 26.3 \mathrm{~nm}$ \\
$\mathrm{PSN}-\mathrm{Ru}$ & $\mathrm{PbSc}_{0.507} \mathrm{Nb}_{0.469} \mathrm{Ru}_{0.024} \mathrm{O}_{3}$ & $P m \overline{3} m, a=4.0787(2) \AA$ & $\begin{array}{c}\mathrm{N} \\
\text { PZN-0.1PT-Ru }\end{array}$ \\
\hline \hline
\end{tabular}

a Determined from powder XRD as $\rho_{\text {sample }} / \rho_{\text {fully ordered }}, \rho=I(111) / I(200)$, according to Ref. [29].

${ }^{b}$ From the Scherrer equation applied to the 111 Bragg reflection in the power XRD patterns.

\section{B. X-ray absorption spectroscopy}

Room-temperature XAS measurements were performed at the $K$ absorption edge ( $K$ edge) of $\mathrm{Ru}(22117 \mathrm{eV})$ in PST-Ru, PSN-Ru, PZN-0.1PT-Ru, the $L_{3}$ edge of Ta $(9881 \mathrm{eV})$ in PST-Ru, and the $K$ edge of $\mathrm{Nb}(18986 \mathrm{eV})$ in PSN-Ru at the XAS-Beamline at the ANKA synchrotron radiation facility, KIT, Karlsruhe, Germany. A double-crystal monochromator (DCM) equipped with a set of $\mathrm{Si}(311)$ crystals was employed for energy monochromatization. The samples were one-sided polished $\{100\}$-oriented cuts of equal thickness attached next to each other onto a Kapton tape. All studied oriented single crystals are cubic or multidomain pseudocubic, which eliminates polarization-dependent effects in the XAS data. Commercially available powders of high-purity $\mathrm{RuCl}_{3}$ and $\mathrm{RuO}_{2}$ (Alfa Aesar) as well as in-house synthesized single crystals of $\mathrm{Pb}_{2} \mathrm{Ru}_{2} \mathrm{O}_{6.5}$ and $\mathrm{Ca}_{2} \mathrm{YRuO}_{6}$ were used as reference materials for $\mathrm{Ru}^{3+}, \mathrm{Ru}^{4+}, \mathrm{Ru}^{4.5+}$, and $\mathrm{Ru}^{5+}$, respectively. The structure and chemical composition of $\mathrm{Pb}_{2} \mathrm{Ru}_{2} \mathrm{O}_{6.5}$ and $\mathrm{Ca}_{2} \mathrm{YRuO}_{6}$ were verified by XRD and electron microprobe analysis. XAS data of $\mathrm{Pb}_{2} \mathrm{Ru}_{2} \mathrm{O}_{6.5}$, which has a cubic symmetry, were also collected from $\{100\}$-oriented cuts fixed on a Kapton tape, whereas the crystals of $\mathrm{Ca}_{2} \mathrm{YRuO}_{6}$, which exhibit a strong structural anisotropy, were powdered. Each powder reference sample was mixed with $100 \mathrm{mg} \mathrm{BN}$ and pressed in a pellet with a diameter of $13 \mathrm{~mm}$. The XAS (XANES+EXAFS) data of the reference pellets and crystalline samples were collected in transmission and fluorescence mode, respectively. A five-element Ge solid state detector (Canberra LEGe type) was used for all measurements in fluorescence mode. At least six XAS spectra were collected from the compounds measured in fluorescence mode in order to check the reproducibility of the spectral features and to improve the counting statistics. The DCM was calibrated by assigning $24350 \mathrm{eV}$ to the first inflection point of a $K$-edge XANES spectrum of a Pd foil. A repeatedly measured spectrum of $\mathrm{Pb}_{2} \mathrm{Ru}_{2} \mathrm{O}_{6.5}$ was used for calibration of the XAS spectra.

The ATHENA and ARTEMIS program packages, part of the IFFEFIT software package [33], were used for data reduction of the XAS spectra and modeling of the EXAFS spectra, respectively. The scattering paths used to fit the EXAFS spectra were generated by the FEFF8.2 theoretical code based on the multiple scattering theory [34]. The Fermi energy was accurately calculated by using the self-consistent field (SCF) card implying self-consistent calculation of the atomic scattering potentials. A cubic face-centered structure $(F m \overline{3} m)$ with a unit-cell parameter $2 a$ determined by XRD (Table I) and occupied Wyckoff positions: $8 c(0.25,0.25,0.25)$ for the $A$-site cations, $4 a(0,0,0)$ and $4 b(0.5,0.5,0.5)$ for the $B$-site cations, and $24 e(0.5,0,0)$ for $\mathrm{O}$, were used to generate the atomic coordinates in the initial structural models for all three relaxor samples. Simulations with different orientations of the crystal with respect to the polarization of the beam were performed to verify the absence of any polarization-dependent effects on the XAS data. The EXAFS signals covering $k$ ranges specified in Table II were Fourier transformed to $R$ space using a $k$ weighting of 1,2 , or 3 and a Hanning window with window sills $d k$ equal to 2. Fits were performed in the $R$ space over $R$ ranges given in Table II. The amplitude reduction factor $S_{0}^{2}$ calculated with the FEFF8.2 code was held constant to 1 . The ARTEMIS option for simultaneous fits with all three $k$ weightings $(1,2$, and 3$)$ was used. We have used a shell-by-shell fitting approach in which first, the FT-EXAFS peak at the shortest $R$ distance was modeled by considering the nearest coordination sphere ( $\mathrm{O}$ atoms) and then, the longer-distance FT-EXAFS spectral range was modeled by including single scattering from the $\mathrm{Pb}$ coordination sphere as well as the $B$-site cation coordination sphere. The fittings were initially performed with single scattering paths only, which resulted in a goodness of fit below 0.015 for all analyses (see Table II). Although this already indicates a rather good agreement between data and fits $(1.5 \%$ on average or better), we considered as a next step multiple scattering paths. Different scattering models were implemented but the contribution from multiple scattering paths in the EXAFS spectra of relaxors was either negligible or yielded physically self-inconsistent results (see the Supplemental Material [35] for details.). Hence, the results from the EXAFS fits reported here are based only on single scattering paths listed in the first column of Tables III and IV.

It should be mentioned that due to the existence of dynamic $B$-site cation off-center displacements in relaxors, one can expect multiple distances and consequently an asymmetric peak shape in the corresponding EXAFS spectra, instead of

TABLE II. Covered $k$ ranges and $R$ ranges as well as the goodness of fits ( $R$ factor) in the preformed fittings of EXAFS spectra.

\begin{tabular}{lccc}
\hline \hline Data set & $k$ range $\left(\AA^{-1}\right)$ & $R$ range $(\AA)$ & $R$ factor \\
\hline Ru $K$ edge, PST-Ru & $3.5-9.3$ & $1.0-4.0$ & 0.002 \\
Ru $K$ edge, PSN-Ru & $3.5-11.3$ & $1.0-4.0$ & 0.010 \\
Ru $K$ edge, PZN-0.1PT-Ru & $3.6-9.9$ & $0.8-4.4$ & 0.008 \\
Ta $L_{3}$ edge, PST-Ru & $2.6-10.6$ & $1.0-3.8$ & 0.014 \\
$\mathrm{Nb} K$ edge, PSN-Ru & $3.6-9.3$ & $1.0-3.8$ & 0.002 \\
\hline \hline
\end{tabular}


TABLE III. Distances $d$ to neighboring atoms, coordination numbers $N$, mean-square displacement (Debye-Waller factors) $\sigma^{2}$, and shift of the ionization potential $\Delta E_{0}$ for Ru and the major $B^{\prime \prime}$-site cation in PST-Ru and PSN-Ru obtained from EXAFS analysis as well as the corresponding distances and angles in a face-centered-cubic $A B^{\prime}{ }_{0.5} B^{\prime \prime}{ }_{0.5} \mathrm{O}_{3}$ structure with a unit-cell parameter $2 a$ determined by XRD; $A=\mathrm{Pb}$, $B^{\prime}=\mathrm{Sc}, B^{\prime \prime}=\mathrm{Ta}$ or $\mathrm{Nb}$. See the Supplemental Material [35] for more details on the various scattering models used in the EXAFS analysis.

\begin{tabular}{|c|c|c|c|c|c|c|c|c|}
\hline & \multicolumn{4}{|c|}{ PST-Ru } & \multicolumn{4}{|c|}{ PSN-Ru } \\
\hline & $d(\AA)$ & $N$ & $\sigma^{2}\left(\AA^{2}\right)$ & $\Delta E_{0}(\mathrm{eV})$ & $d(\AA)$ & $N$ & $\sigma^{2}\left(\AA^{2}\right)$ & $\Delta E_{0}(\mathrm{eV})$ \\
\hline$\left(B^{\prime \prime}-O\right)_{\text {cubic }}=a / 2$ & 2.03375 & 6 & & & 2.03935 & 6 & & \\
\hline $\mathrm{Ru}-\mathrm{O}$ & $1.97 \pm 0.01$ & $4.6 \pm 0.3$ & $0.0030(2)$ & $-5.7 \pm 0.3$ & $1.97 \pm 0.01$ & $5.5 \pm 0.4$ & $0.0060(4)$ & $-7.9 \pm 0.4$ \\
\hline $\mathrm{Ta}-\mathrm{O}$ & $1.96 \pm 0.01$ & $4.3 \pm 0.4$ & $0.0040(7)$ & $1.6 \pm 0.5$ & & & & \\
\hline $\mathrm{Nb}-\mathrm{O}$ & & & & & $1.95 \pm 0.01$ & $5.6 \pm 0.6$ & $0.0100(3)$ & $-9.9 \pm 0.3$ \\
\hline$\left(B^{\prime \prime}-A\right)_{\text {cubic }}=a \sqrt{3} / 2$ & 3.5225 & 8 & & & 3.5322 & 8 & & \\
\hline $\mathrm{Ru}-\mathrm{Pb}$ & $3.30 \pm 0.03$ & $2.9 \pm 1.6$ & $0.010(1)$ & $-6.8 \pm 1.6$ & $3.30 \pm 0.04$ & $3.6 \pm 2.4$ & $0.010(4)$ & $-3.4 \pm 1.8$ \\
\hline $\mathrm{Ta}-\mathrm{Pb}$ & $3.47 \pm 0.02$ & $7.8 \pm 2.3$ & $0.0040(15)$ & $-3.7 \pm 0.8$ & & & & \\
\hline $\mathrm{Nb}-\mathrm{Pb}$ & & & & & $3.09 \pm 0.02$ & $0.6 \pm 0.4$ & $0.0050(5)$ & $5.6 \pm 0.3$ \\
\hline$\left(B^{\prime \prime}-B^{\prime}\right)_{\text {cubic }}=a$ & 4.0675 & 6 & & & 4.0787 & 6 & & \\
\hline $\mathrm{Ru}-\mathrm{Sc}$ & $3.92 \pm 0.01$ & $5.6 \pm 1.1$ & $0.0060(8)$ & $7.3 \pm 0.4$ & $3.89 \pm 0.03$ & $4.9 \pm 1.4$ & $0.006(1)$ & $7.3 \pm 0.4$ \\
\hline Ta-Sc & $3.77 \pm 0.02$ & $7.0 \pm 2.0$ & $0.0020(14)$ & $4.3 \pm 0.6$ & & & & \\
\hline $\mathrm{Nb}-\mathrm{Sc}$ & & & & & $3.84 \pm 0.01$ & $3.6 \pm 0.7$ & $0.0050(5)$ & $5.6 \pm 0.3$ \\
\hline$B^{\prime}-\mathrm{O}\left(B^{\prime \prime}\right)_{\text {cubic }}=a / 2$ & 2.03375 & & & & 2.03935 & & & \\
\hline $\mathrm{Sc}-\mathrm{O}(\mathrm{Ru})=a-(\mathrm{Ru}-\mathrm{O})$ & $2.09 \pm 0.01$ & & & & $2.11 \pm 0.01$ & & & \\
\hline $\mathrm{Sc}-\mathrm{O}(\mathrm{Ta})=a-(\mathrm{Ta}-\mathrm{O})$ & $2.11 \pm 0.01$ & & & & & & & \\
\hline $\mathrm{Sc}-\mathrm{O}(\mathrm{Nb})=a-(\mathrm{Nb}-\mathrm{O})$ & & & & & $2.13 \pm 0.01$ & & & \\
\hline & $\theta=\angle\left(B^{\prime}-\mathrm{O}-B^{\prime \prime}\right)^{\mathrm{a}}$ & & Tilt $\varphi=(180-\theta) / 2$ & & $\theta=\angle\left(B^{\prime}-\mathrm{O}-B^{\prime \prime}\right)^{\mathrm{a}}$ & & Tilt $\varphi=(180-\theta) / 2$ & \\
\hline$\left(B^{\prime}-\mathrm{O}-B^{\prime \prime}\right)_{\text {cubic }}$ & $180^{\circ}$ & & $0^{\circ}$ & & $180^{\circ}$ & & $0^{\circ}$ & \\
\hline $\mathrm{Sc}-\mathrm{O}-\mathrm{Ru}$ & $144^{\circ} \mathrm{b} / 149^{\circ \mathrm{c}}$ & & $18^{\circ \mathrm{b}} / 15.5^{\circ \mathrm{c}}$ & & $145^{\circ \mathrm{b}} / 146^{\circ \mathrm{c}}$ & & $17.5^{\circ} \mathrm{b} / 17^{\circ \mathrm{c}}$ & \\
\hline Sc-O-Ta & $133^{\circ} \mathrm{b} / 136^{\circ} \mathrm{c}$ & & $23.5^{\circ} \mathrm{b} / 22^{\circ \mathrm{c}}$ & & & & & \\
\hline $\mathrm{Sc}-\mathrm{O}-\mathrm{Nb}$ & & & & & $141^{\circ \mathrm{b}} / 143^{\circ \mathrm{c}}$ & & $19.5^{\circ \mathrm{b}} / 18.5^{\circ \mathrm{c}}$ & \\
\hline
\end{tabular}

${ }^{\mathrm{a}} \theta=\arccos \left\{\left[\left(B^{\prime}-\mathrm{O}\right)^{2}+\left(B^{\prime \prime}-\mathrm{O}\right)^{2}-\left(B^{\prime}-B^{\prime \prime}\right)^{2}\right] /\left[2\left(B^{\prime}-\mathrm{O}\right)\left(B^{\prime \prime}-\mathrm{O}\right)\right]\right\}$.

${ }^{\mathrm{b}}$ Calculated with $B^{\prime \prime}-\mathrm{O}$ and $B^{\prime}-B^{\prime \prime}$ obtained from EXAFS, and $B^{\prime}-\mathrm{O}=a_{\mathrm{XRD}}-\left(B^{\prime \prime}-\mathrm{O}\right)_{\mathrm{EXAFS}}$.

${ }^{c}$ Calculated with $B^{\prime \prime}-\mathrm{O}$ and $B^{\prime}-B^{\prime \prime}$ obtained from EXAFS and $B^{\prime}-\mathrm{O}=2.104 \AA$, which is the experimentally determined Sc-O distance in pure PST from Sc $K$-edge EXAFS analysis [19].

the commonly used Gaussian peak shape. An indicator for significant $B$-cation hopping and multiple distances are the Debye-Waller factors, which should be considerably larger than 0.01-0.02 $\AA^{2}$, when fittings are performed with Gaussian functions [36]. In our case the obtained Debye-Waller factors are less than $0.01 \AA^{2}$, indicating that the $B$-cation off-centering is less than $0.1 \AA$. This value is in good agreement with

TABLE IV. Distances $d$ to neighboring atoms and coordination numbers $N$, mean-square displacement (Debye-Waller factors) $\sigma^{2}$, and shifts of the ionization potential $\Delta E_{0}$ for Ru in PZN-PT-Ru as well as the main distances in a primitive cubic $A B \mathrm{O}_{3}$ structure with a unit-cell parameter $a$ determined by XRD; $A=\mathrm{Pb}, B=\mathrm{Zn}$, Nb, Ti.

\begin{tabular}{lcccc}
\hline \hline & \multicolumn{4}{c}{$\mathrm{PZN}-\mathrm{PT}-\mathrm{Ru}$} \\
\cline { 2 - 5 } & $d(\AA)$ & $N$ & $\sigma^{2}\left(\AA^{2}\right)$ & $\Delta E_{0}(\mathrm{eV})$ \\
\hline $\mathrm{Ru}-\mathrm{O}$ & $2.02 \pm 0.01$ & $5.5 \pm 0.7$ & $0.0070(6)$ & $6.8 \pm 0.5$ \\
$\mathrm{Ru}-\mathrm{Zn}$ & $2.97 \pm 0.02$ & $1.2 \pm 0.4$ & $0.0070(6)$ & $8.7 \pm 0.3$ \\
$\mathrm{Ru}-\mathrm{Ru}$ & $3.40 \pm 0.02$ & $2.0 \pm 0.8$ & $0.0070(6)$ & $8.7 \pm 3.8$ \\
$\mathrm{Ru}-\mathrm{Pb}$ & $4.00 \pm 0.02$ & $5.7 \pm 1.7$ & $0.0070(6)$ & $6.8 \pm 0.5$ \\
$(B-\mathrm{O})_{\text {cubic }}=a / 2$ & 2.02605 & 6 & & \\
$(A-\mathrm{O})_{\text {cubic }}=a \sqrt{2} / 2$ & 2.86527 & 12 & & \\
$(B-A)_{\text {cubic }}=a \sqrt{3} / 2$ & 3.50922 & 8 & & \\
$(B-B)_{\text {cubic }}=a$ & 4.0521 & 6 & & \\
$(A-A)_{\text {cubic }}=a$ & 4.0521 & 6 & & \\
\hline \hline
\end{tabular}

the values of $B$-cation off-center displacements predicted by density functional theory (DFT) calculations [24] but it is on the limit of the distance resolution of EXAFS $(\Delta R \sim 1 / \Delta k)$. In addition, we have calculated the possible $B-B$ distances within the framework of the eight-state Potts model [37] and performed statistical analysis. If the cubic unit cell is $4.07 \AA$ and the $B$-site off-centering is $0.1 \AA$, the mean $B$ - $B$ distance is $4.0718 \AA$, whereas the median is $4.0716 \AA$, i.e., they are essentially the same and hence one should expect a peak shape very close to a Gaussian function, i.e., there are no indications that the use of symmetric peak-shape functions is insufficient and that more sophisticated asymmetric peak-shape functions should be implemented to model the EXAFS spectra presented here.

\section{RESULTS AND DISCUSSION}

\section{A. Synchrotron XRD, Raman scattering, and optical spectroscopy}

The existence of polar nanoregions in $\mathrm{Pb}$-based perovskitetype relaxors can be revealed by the $\mathrm{x}$-ray diffuse scattering along the cubic $\langle 110\rangle^{*}$ direction of the reciprocal space [38], resulting in a specific "butterfly" or elliptic shape of the observed $h k l$ Bragg reflections with $h, k, l$, all even (Miller indices are given in $F m \overline{3} m$ throughout the paper). Recent three-dimensional mapping of this X-ray diffuse scattering [39] 
emphasized that the polar nanoregions should be considered as spatial regions of dynamically coupled polar distortions surrounded by a pseudoparaelectric matrix containing uncoupled local structural distortions rather than static entries with a polar rhombohedral structure that are insufficiently large to produce sharp Bragg spots. As can be seen in Fig. 1, both PST-Ru and PSN-Ru contain x-ray diffuse scattering near room temperature $(300 \mathrm{~K})$, indicating that both compounds are in a relaxor state, similar to the corresponding pure compounds [41,42]. Dielectric permittivity measurements on PST-Ru using a probe frequency of $10 \mathrm{kHz}$ revealed a broad maximum at $T_{m} \sim 260 \mathrm{~K}$. At $150 \mathrm{~K}$ the butterfly-shaped and elliptic $\mathrm{x}$-ray diffuse scattering still persist for PST-Ru and PSN-Ru, whereas it is vanished for PST and PSN on account of formation of a polar rhombohedral phase. This indicates that the incorporation of $\mathrm{Ru}$ into the relaxor structure hinders the development of long-range ferroelectric order on cooling. The same trend was observed for Ru-doped PZN-0.1PT [23]. It should be, however, emphasized that although all three Rudoped compounds studied here exhibit x-ray diffuse scattering at room temperature (Fig. 1 and Ref. [23]), indicating that they all are in a relaxor state, according to XRD PST-Ru and PSN-Ru are still the high-temperature cubic phase at room temperature, whereas PZN-PT-Ru already exhibits a ferroelectric long-range order which is predominantly monoclinic [23].

The Raman spectra of PST-Ru and PSN-Ru measured with an excitation wavelength $\lambda=514.5 \mathrm{~nm}$ considerably differ from the Raman scattering of the corresponding pure compounds (see Fig. 2). The Raman scattering near $720 \mathrm{~cm}^{-1}$ is strongly enhanced. This Raman peak originates from an antisymmetric $\mathrm{BO}_{6}$ stretching mode in polar nanoregions [43], which is related to the longitudinal component of an infraredactive $F_{1 u}$ mode in the cubic structure. The corresponding second-order Raman scattering near $1440 \mathrm{~cm}^{-1}$ is also enhanced for Ru-doped PST and PSN as compared to the pure compounds (see Fig. 2). Such a simultaneous enhancement of the first- and second-order Raman scattering related to internal antisymmetric $\mathrm{BO}_{6}$ vibrations is observed in resonance Raman spectra excited with $\lambda=325 \mathrm{~nm}\left(E_{\text {incident photon }}=3.8 \mathrm{eV}>E_{g}\right)$ and it is related to the presence of noncentrosymmetric (polar) atomic arrangements [44]. On the other hand, the Raman scattering of Ru-doped PST and PSN excited with $325 \mathrm{~nm}$ resembles that of pure PST and PSN (Fig. 2) but differs from the Raman spectra of PST-Ru and PSN-Ru measured with $514.5 \mathrm{~nm}$. Therefore the unusual Raman scattering excited with $\lambda=514.5 \mathrm{~nm}\left(E_{\text {incident photon }}=2.4 \mathrm{eV}\right)$ suggests a significant change in the energy gap due to local distortions induced by the incorporation of Ru into the PST and PSN relaxor matrices. Indeed, optical spectroscopic data on PST and PST-Ru revealed that Ru doping leads to a red shift of the optical absorption edge, which corresponds to a reduction of $E_{g}$ related to direct electron transitions by $1 \mathrm{eV}$ (Fig. 3). In addition, the Raman spectrum of PST-Ru measured with $\lambda=1064 \mathrm{~nm}\left(E_{\text {incident photon }}=1.2 \mathrm{eV}<E_{g}\right)$ resembles the nonresonance Raman scattering of PST (Fig. 3); the main Ruinduced changes are the weakening of the Raman scattering near $350 \mathrm{~cm}^{-1}$ and the slight enhancement of the Raman scattering near $720 \mathrm{~cm}^{-1}$. The former has been observed for all studied $A$ - or $B$-site doped PST and PSN [41-43,45] and indicates disturbance of coherent $\mathrm{Pb}-\mathrm{O}$ ferroic species. A very
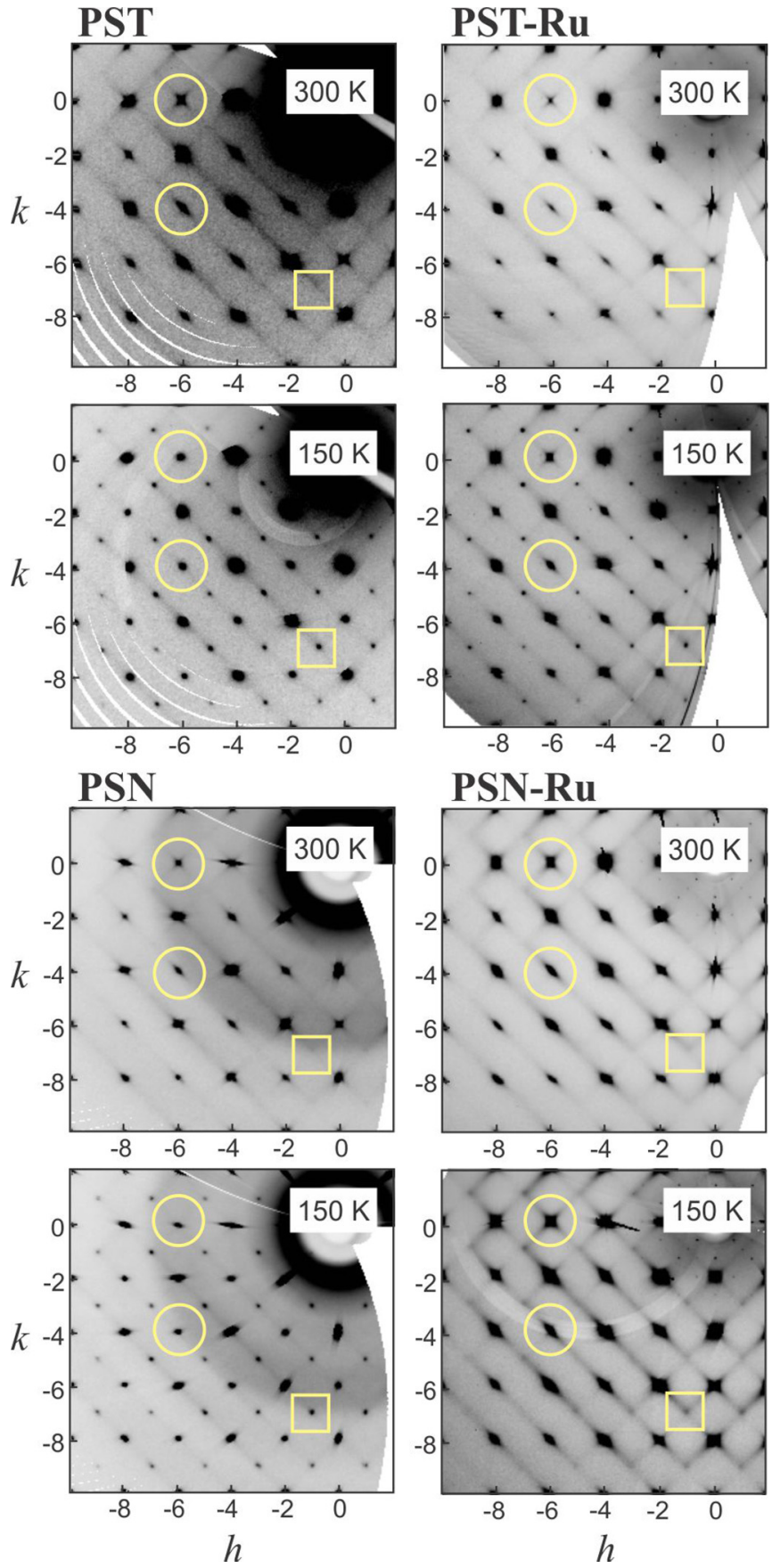

FIG. 1. (Color online) $h k 0$ layers of pure and Ru-doped PST and PSN reconstructed from synchrotron single-crystals XRD measured at 300 and $150 \mathrm{~K}$; Miller indices are given in $F m \overline{3} m$. The open circles mark representative Bragg reflections which in a relaxor state

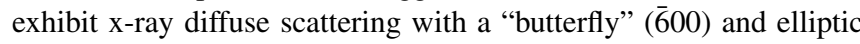
$(\overline{6} \overline{4} 0)$ shapes typical of polar nanoregions in $\mathrm{Pb}$-based perovskitetype relaxors. The open squares mark odd-odd-even $h k l$ points with $h, k$ odd, and $l$ even, which show diffraction peaks when a polar rhombohedral double-perovskite phase $R 3$ is formed [40].

weak doping-induced enhancement of the Raman scattering near $720 \mathrm{~cm}^{-1}$ was observed for PST heavily doped with $\mathrm{Sn}^{4+}$ and it was attributed to shifts of the $B$-positioned four-valent dopant along the cubic $\langle 001\rangle$, similar to the $B$-site cation in $\mathrm{PbTiO}_{4}$ [43]. Therefore, one can suggest that $\mathrm{Ru}$ incorporated 
excitation wavelength $\lambda=514.5 \mathrm{~nm}$

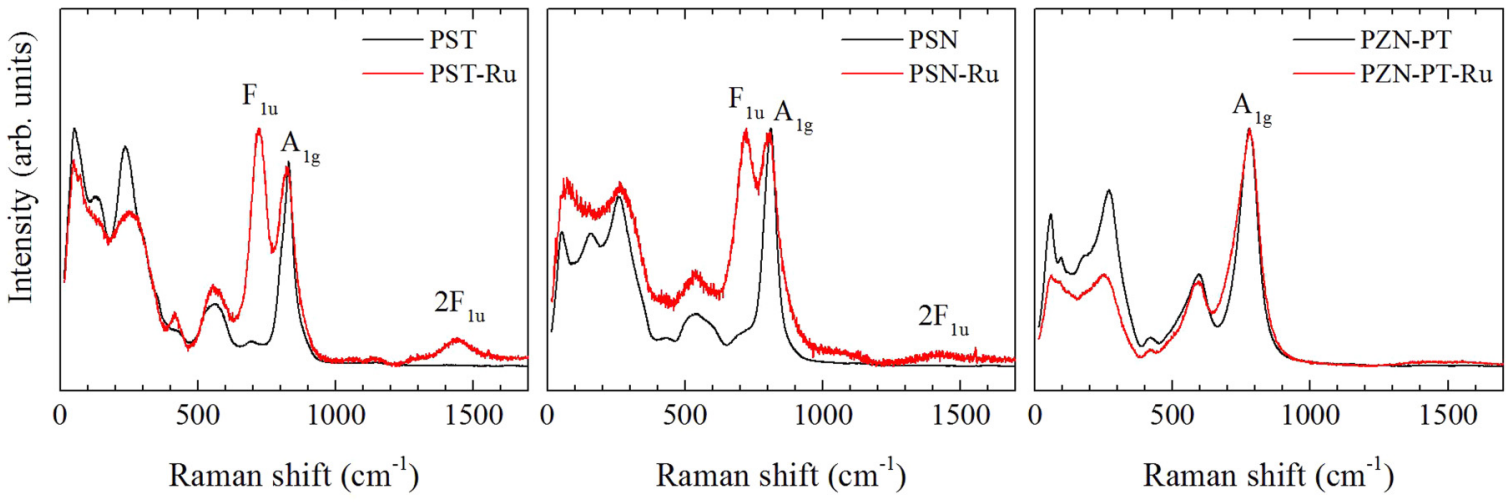

excitation wavelength $\lambda=325 \mathrm{~nm}$

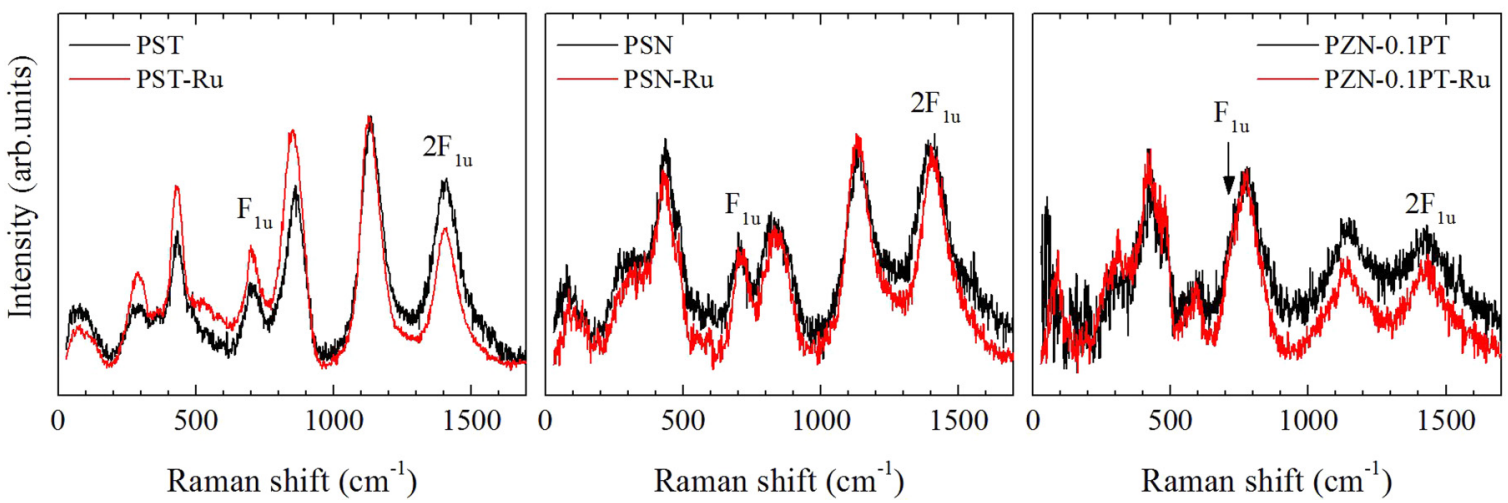

FIG. 2. (Color online) Room-temperature $\bar{Z}(X X) Z$ Raman spectra of pure (black lines) and Ru-doped (red lines) PST, PSN, and PZN-0.1PT excited with a laser wavelength of $514.5 \mathrm{~nm}$ (upper plots) and $325 \mathrm{~nm}$ (bottom plots); $X$ and $Z$ are along the cubic [100] and [001] directions. $F_{1 u}$ and $A_{1 g}$ mark the antisymmetric and symmetric $B \mathrm{O}_{6}$ stretching mode, respectively, which in a cubic $A B \mathrm{O}_{3}$ compound give rise correspondingly to an infrared- and Raman-active crystal normal mode.

into $\mathrm{Pb} B^{\prime}{ }_{0.5} B^{\prime \prime}{ }_{0.5} \mathrm{O}_{3}$ also leads to such local structural changes. The strong resonance enhancement of the Raman scattering near $720 \mathrm{~cm}^{-1}$ with $\lambda=514.5 \mathrm{~nm}$ for PST-Ru and PSN-Ru, regardless of the low dopant concentration, implies the idea of Ru-induced electron-phonon coupling and associated JahnTeller octahedral distortions.

As can be seen in Fig. 2, the Raman spectrum of Ru-doped PZN-0.1PT measured with $\lambda=514.5 \mathrm{~nm}$ resembles that of PZN-0.1PT, i.e., no resonance enhancement of the Raman scattering is observed. Optical spectroscopy revealed that the energy gap of direct electron transitions is $\sim 3.1 \mathrm{eV}$ for PZN$0.1 \mathrm{PT}$ and $\sim 2.7 \mathrm{eV}$ for PZN-0.1PT-Ru, i.e., Ru doping has a considerably less effect on the electron properties of PZN0.1PT as compared to PST and PSN, which may be due to the less dopant concentration and/or to the different way of incorporation of $\mathrm{Ru}$ into the relaxor host matrix. Thus in order to elucidate the way of Ru incorporation into PST, PSN, and PZN-0.PT, we have performed XAS investigations.

\section{B. XANES analysis}

Figure 4 shows the $\mathrm{Ru} K$-edge XAS spectra of PST$\mathrm{Ru}, \mathrm{PSN}-\mathrm{Ru}$, and PZN-01.PT-Ru along with the spectra of reference compounds for $\mathrm{Ru}^{3+}\left(\mathrm{RuCl}_{3}\right), \mathrm{Ru}^{4+}\left(\mathrm{RuO}_{2}\right), \mathrm{Ru}^{4.5+}$ $\left(\mathrm{Pb}_{2} \mathrm{Ru}_{2} \mathrm{O}_{6.5}\right)$, and $\mathrm{Ru}^{5+}\left(\mathrm{Ca}_{2} \mathrm{YRuO}_{6}\right)$. The overall resonance shapes of the postedge features of the PST-Ru and PSN-Ru are very similar [see Fig. 4(a)], whereas the XAS spectrum of the PZN-01.PT-Ru exhibits a considerably different spectral shape, which is also revealed in the results obtained from in the EXAFS analyses given below. The core hole created during the XAS process can be described as an insertion of a positive charge close to the nucleus. The level of redistribution of electronic density due to screening of this charge depends strongly on the oxidation state of the probed atom and it is correlated to the energy position of the absorption edge. As can be seen from the enlarged-scale plots, the $K$ edge of PZN-0.1PT-Ru is positioned between those of $\mathrm{RuCl}_{3}$ and $\mathrm{RuO}_{2}$ [Fig. 4(b)], whereas the $K$ edges of PST-Ru and PSN-Ru are nearly the same as that of $\mathrm{Pb}_{2} \mathrm{Ru}_{2} \mathrm{O}_{6.5}$ [Fig. 4(c)]. This suggests that the average oxidation state of Ru in PZN-0.1PT is between 3+ and 4+, while that of Ru in PST and PSN is close to $4.5+$. One empirical method to determine the average oxidation state of $\mathrm{Ru}^{n+}$ is shown in Fig. 5. Following the commonly established approach, we determined the position of the x-ray absorption edge $E_{0}$ from the maximum of the energy derivative of the X-ray absorption coefficient $\mu(E)$ (see Fig. 5, left), which corresponds to the inflection point of the absorption edge. The peak positions of $d \mu(E) / d E$ were derived from Gaussian fits to the experimental curves. Then, we plotted the mean oxidation state of the measured reference materials versus $\Delta E=E_{0}^{\text {sample }}-E_{0}^{\text {metal Ru }}$, where $E_{0}^{\text {metal Ru }}=$ $22117 \mathrm{eV}$ is the tabulated $\mathrm{Ru} K$ edge of metallic $\mathrm{Ru}$, and 

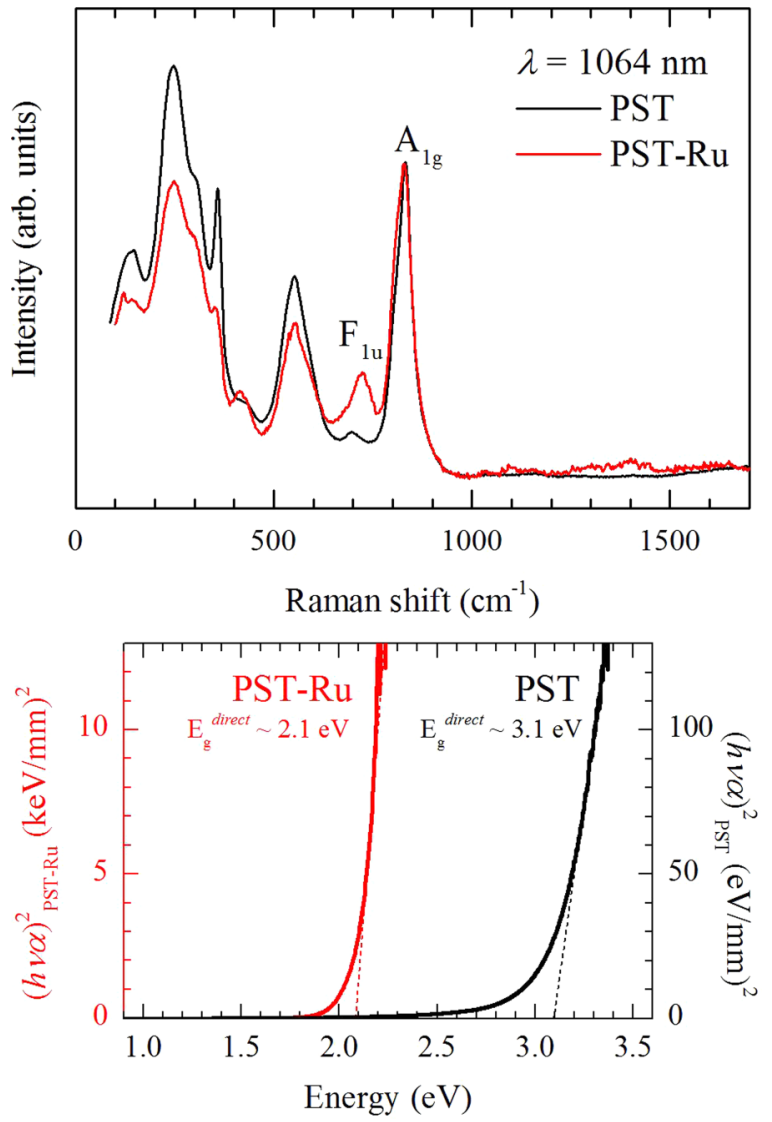

FIG. 3. (Color online) Room-temperature unpolarized Raman spectra of pure and Ru-doped PST excited with a near-infrared laser wavelength of $1064 \mathrm{~nm}$ (upper plot) as well as the square of the product of the optical absorption coefficient $\alpha$ and the photon energy $E$ versus the photon energy $E$ for PST and PST-Ru (bottom plot); the dashed lines represent linear fits to the corresponding slopes of $(h v \alpha)^{2}$ as a function of $E$.

a linear fit to the data points was performed (Fig. 5, right). Using the linear function between the oxidation state and the shift of the Ru $K$ edge $\Delta E$, the mean oxidation state $n$ of $\mathrm{Ru}^{n+}$ incorporated into PZN-0.1PT was calculated to be $n=3.8 \pm$ 0.1 , whereas $n=4.4 \pm 0.1$ for PST-Ru and PSN-Ru. The XANES results on PZN-01.PT-Ru indicate that the majority of $\mathrm{Ru}$ has an oxidation state of 4 , with a minor content of $\mathrm{Ru}$ that has an average oxidation state lower than 4 . This is in good accordance with a previous electron paramagnetic resonance analysis of PZN-0.1PT-Ru [15] revealing the existence of paramagnetic $\mathrm{Ru}^{3+}$ and $\mathrm{Ru}^{5+}$ centers, with the fraction of $\mathrm{Ru}^{3+}$ being larger than the fraction of $\mathrm{Ru}^{5+}$ at room temperature.

\section{EXAFS analysis}

Deeper insights into the atomic arrangements on the short and intermediate scale can be gained from EXAFS spectra, by considering the $k$-weighted $\chi(k)$ oscillatory part obtained from $\chi(E)=\frac{\mu(E)-\mu_{0}(E)}{\Delta \mu_{0}\left(E_{0}\right)}$, where $k=\sqrt{2 m\left(E-E_{0}\right) / \hbar^{2}}$ is the photoelectron wave number, $\mu_{0}(E)$ is a background function, and $\Delta \mu_{0}\left(E_{0}\right)$ is the absorption edge step at $E_{0}$, as well as its Fourier transform $\operatorname{FT}\left[\chi(k) k^{w}\right]$ in the $R$ space $(w=$
1,2 , or 3) and the subsequent back Fourier transform of FT $\left[\chi(k) k^{w}\right]$ denoted here as $\chi(q) q^{w}$. Several models were implemented to fit the experimental FT-EXAFS spectra: $\mathrm{Ru}$ on the 12-coordinated $A$ site, Ru on the 6-coordinated $B$ site surrounded only by $\mathrm{Sc} / \mathrm{Ta}$ or $\mathrm{Nb}$ (for PST-Ru and PSN-Ru), $\mathrm{Ru}$ on the 6-coordinated $B$ site surrounded only by $\mathrm{Zn} / \mathrm{Nb} / \mathrm{Ti}$ (for PZN-0.1PT-Ru), and $\mathrm{Ru}$ on the 6-coordinated $B$ site surrounded only by $\mathrm{Ru}$.

\section{PST-Ru and PSN-Ru}

The modeling of the Ru $K$-edge EXAFS spectra yielded rather similar results for PST-Ru and PSN-Ru (see the Experimental Details section and the Supplemental Material [35] for details on various scattering models used in the EXAFS analysis). A-positioned $\mathrm{Ru}$ was ruled out due to the large discrepancy between the experimental and modeled spectra, indicating that $\mathrm{Ru}$ entirely substitutes for $B$-site cations in $\mathrm{Pb} B^{\prime}{ }_{0.5} B^{\prime \prime}{ }_{0.5} \mathrm{O}_{3}$ matrices. Models in which the nearest $B$-site neighbors of $\mathrm{Ru}$ are $\mathrm{Ru}$ cations also did not provide reasonable fits to the experimental EXAFS spectra, which excludes the formation of Ru-rich clusters or nanosized phases of the type $\mathrm{PbRuO}_{3}$. The experimental FT-EXAFS spectra of PST-Ru and PSN-Ru at the Ru $K$ edge could be best fitted when a model of $B$-positioned Ru surrounded by $\mathrm{Sc}$ on the nearest $B$ sites was used [see Figs. 6(a)-6(c)]. As can be seen in Figs. 6(a) and $6(\mathrm{~b})$, the modeled curves fit very well to the experimental curves in the entire $k$ and $R$ ranges. The results from the $\mathrm{Ru}$ $K$-edge EXAFS fits of PST-Ru and PSN-Ru unambiguously show that $\mathrm{Ru}$ substitutes for the ferroelectrically active fivevalent $B^{\prime \prime}$ cation in both compounds (see Table III). The fact that the closest $B$-site cations of $\mathrm{Ru}$ are predominantly $\mathrm{Sc}$ cations indicates the presence of chemical 1:1 $B$-site order, consisting of alternating $B^{\prime}$ and $B^{\prime \prime}$ cations along the cubic $\langle 100\rangle$ directions [see the sketch in Fig. 7(a)].

In order to check whether chemical $B$-site order occurs only in the vicinity of $\mathrm{Ru}$ or it is characteristic of the host matrix as well as to compare the atomic surroundings of the Ru dopand and the host $B$-site cations, we analyzed the EXAFS spectra of PST-Ru and PSN-Ru at the corresponding absorption edges of the major $B^{\prime \prime}$ cation: Ta $L_{3}$ edge and $\mathrm{Nb} K$ edge, respectively. Similarly to $\mathrm{Ru}$ the best fits of the corresponding oscillatory parts and their Fourier transforms were obtained when $\mathrm{Ta} / \mathrm{Nb}$ are mostly surrounded by $\mathrm{Sc}$ on the nearest $B$ site [Figs. 6(d) and 6(e)], indicating that $B^{\prime}$-O- $B^{\prime \prime}$ linkages prevail over $B^{\prime \prime}$-O- $B^{\prime \prime}$ linkages, i.e., on the mesoscopic scale PST-Ru and PSN-Ru are chemically ordered, although the degree of long-range chemical order determined by XRD is low or none (see Table I). The same high degree of local chemical $B$-site order, regardless of the very weak long-range chemical order, has been deduced from XAS on $(1-x)$ PST- $x$ PT solid solution [19]. The existence of 1:1 B-site chemical order on the mesoscopic scale leads to a doubling of the unit cell. Hence, the EXAFS results are also consistent with Raman spectra of $\mathrm{Pb}$-based $B$-site complex perovskite-type relaxors, which always exhibit strong Raman peaks near 820 and $50 \mathrm{~cm}^{-1}$ arising from Raman-active modes existing only in a double-perovskite structure, no matter if the structure is cubic or ferroic [23,41-43,45-47]. 

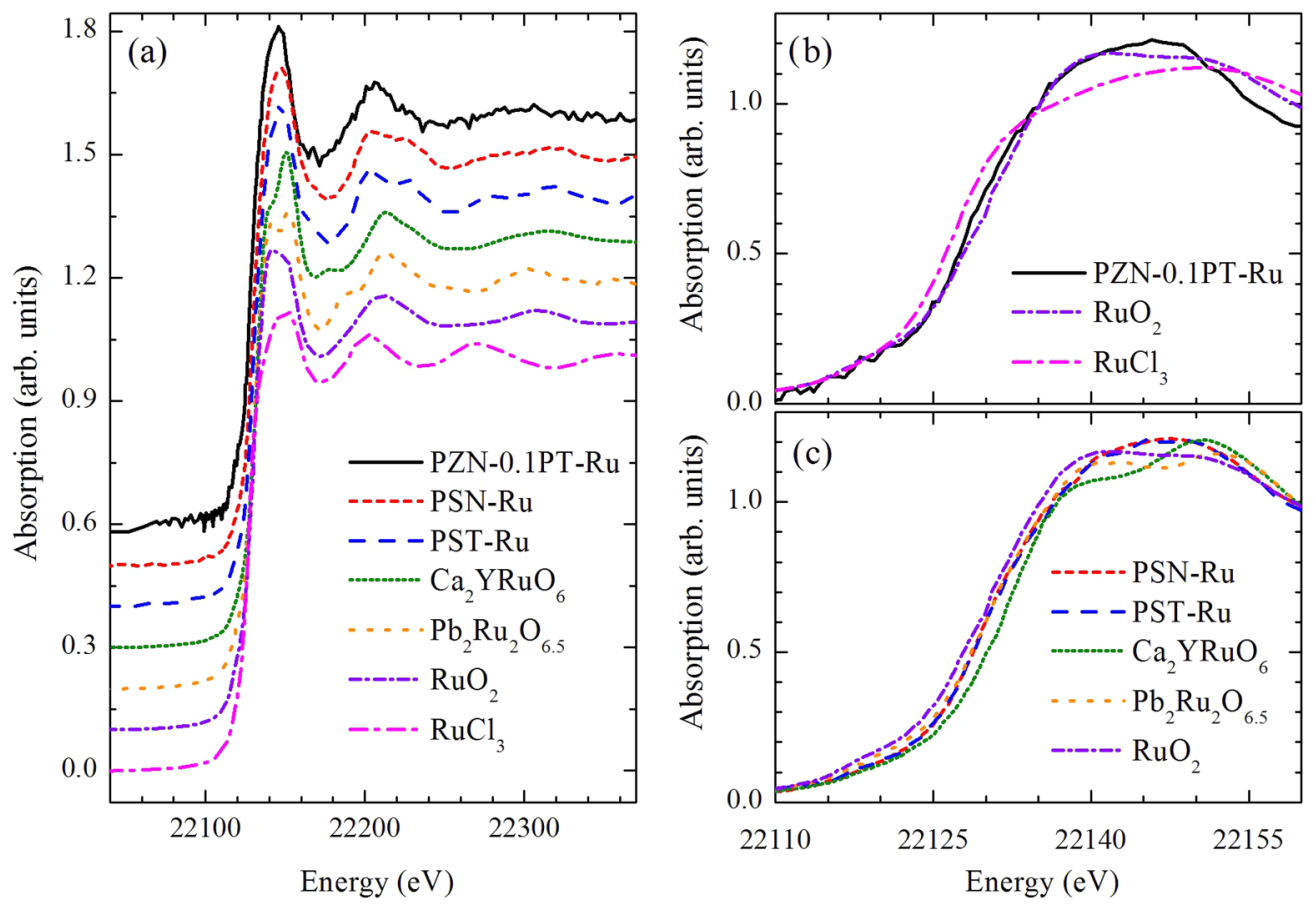

FIG. 4. (Color online) Room-temperature XANES spectra at the Ru $K$ edge of Ru-doped PST, PSN, and PZN-0.1PT as well as of reference compounds for $\mathrm{Ru}^{3+}\left(\mathrm{RuCl}_{3}\right), \mathrm{Ru}^{4+}\left(\mathrm{RuO}_{2}\right), \mathrm{Ru}^{4.5+}\left(\mathrm{Pb}_{2} \mathrm{Ru}_{2} \mathrm{O}_{6.5}\right)$, and $\mathrm{Ru}^{5+}\left(\mathrm{Ca}_{2} \mathrm{YRuO}_{6}\right)$; for clarity the spectra in $4 a$ are vertically off-shifted.

The distances to the neighboring atoms as well as the coordination numbers $N$ for $\mathrm{Ru}, \mathrm{Ta}$, and $\mathrm{Nb}$ derived from the best fits to EXAFS data are summarized in Table III, along with the corresponding interatomic distances and numbers of adjacent atoms in a face-centered-cubic structure $A B^{\prime}{ }_{0.5} B^{\prime \prime}{ }_{0.5} \mathrm{O}_{3}$ with a unit-cell parameter $2 a$ determined by XRD (see Table I). Several important results can be deduced from Table III.

The corresponding distances $\mathrm{Ru}-\mathrm{O} / \mathrm{Pb} / B^{\prime \prime}$ in PST-Ru and $\mathrm{PSN}-\mathrm{Ru}$ are the same within uncertainties, indicating that $\mathrm{Ru}$ enters the relaxor structure of both $\mathrm{Pb} B^{\prime}{ }_{0.5} B^{\prime \prime}{ }_{0.5} \mathrm{O}_{3}$ matrices in the same manner. This is in accordance with almost the same Ru-induced changes in the Raman scattering of PST-Ru and PSN-Ru (see Fig. 2).

Within uncertainties the distances $\mathrm{Ru}-\mathrm{O}$, Ta-O, and Nb$\mathrm{O}$ are the same and they all are slightly shorter than the corresponding value $\left(B^{\prime \prime}-\mathrm{O}\right)_{\text {cubic }}=a_{\mathrm{XRD}} / 2$, suggesting that the oxygen atoms are slightly shifted towards the $B^{\prime \prime}$ cations.
This is consistent with the higher oxidation state of $\mathrm{Ru}^{4.4+}$, $\mathrm{Ta}^{5+}$, and $\mathrm{Nb}^{5+}$ as compared to $\mathrm{Sc}^{3+}$. The independent analysis of the Ta $L_{3}$-edge and Sc $K$-edge EXAFS spectra of $(1-x)$ PST- $x$ PT also revealed that the Ta-O bonds are shorter than the Sc-O bonds and that the sum of Ta-O and $\mathrm{Sc}-\mathrm{O}$ is equal to the unit-cell parameter determined by XRD [19]. Based on the latter result we have calculated the $\mathrm{Sc}-\mathrm{O}\left(B^{\prime \prime}\right)$ distances as $a_{\mathrm{XRD}}-\left(B^{\prime \prime}-\mathrm{O}\right)_{\mathrm{EXAFS}}$ (Table III). Within uncertainties, the Ta-O distance in PST-Ru obtained from the best fit as well as the distance $\mathrm{Sc}-\mathrm{O}(\mathrm{Ta})=a_{\mathrm{XRD}}-(\mathrm{Ta}-\mathrm{O})_{\mathrm{EXAFS}}$ are the same as the experimentally obtained distances Ta-O $=$ $1.961 \pm 0.002 \AA$ and $\mathrm{Sc}-\mathrm{O}=2.104 \pm 0.004 \AA$ for pure PST [19] (Table III). This emphasizes the correctness of the model used here to fit the EXAFS data. For more details on the inconsistency of alternative scattering models see the Supplemental Material [35]. Within uncertainties the obtained coordination number $N$ of the nearest oxygen atoms for $\mathrm{Nb}$
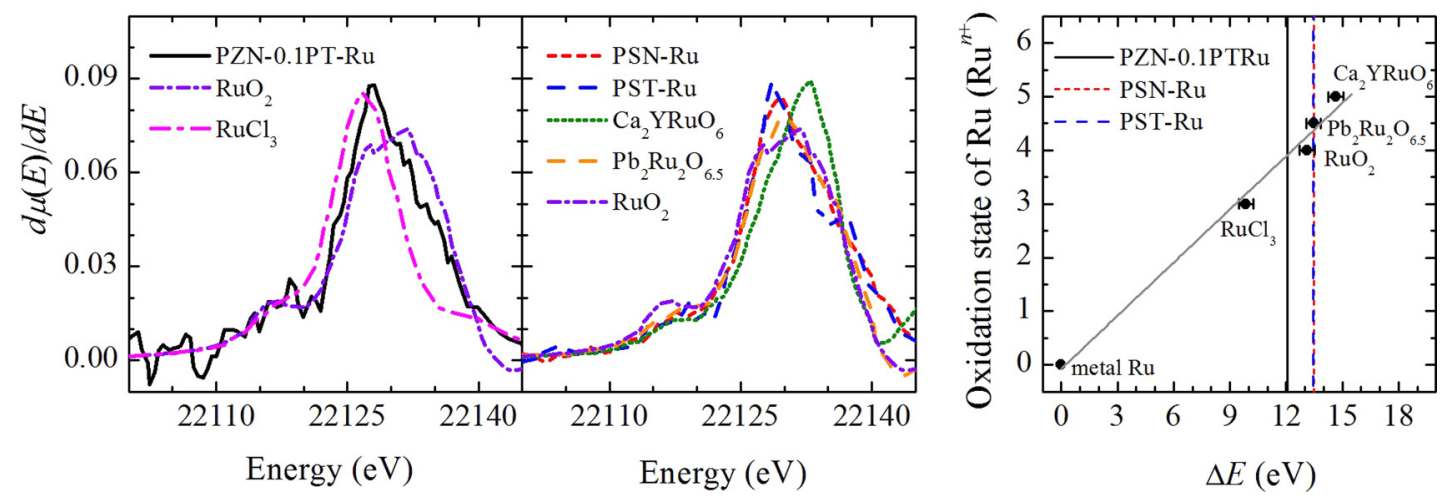

FIG. 5. (Color online) Energy derivative of the normalized absorption $\mu(E)$ (left) and oxidation state of Ru in reference materials versus the shift $\Delta E$ of the Ru $K$ edge with respect to that of metal Ru (right); the gray line represents a linear fit to the data points of reference materials. The vertical lines mark the absorption edge shift for Ru-doped relaxors. 


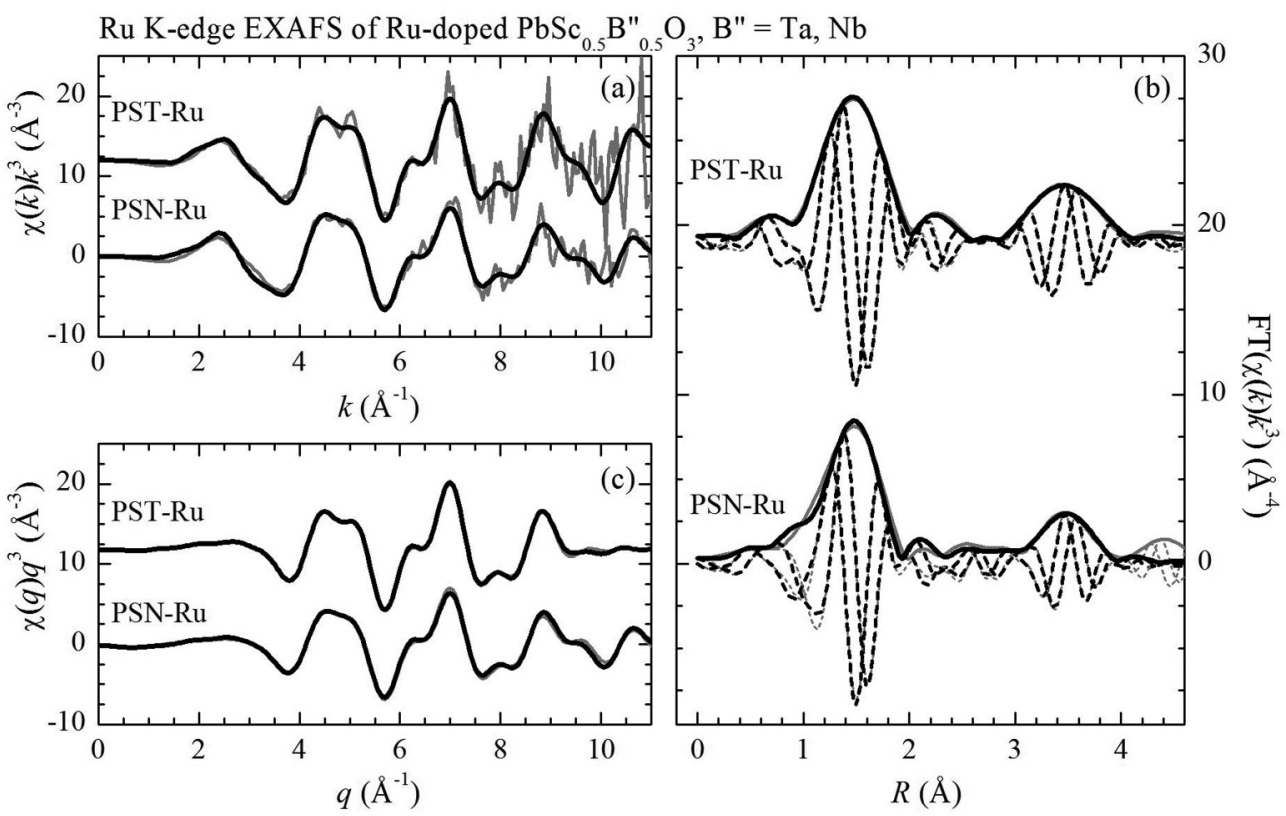

$\mathrm{Ta} \mathrm{L}_{3}$-edge and $\mathrm{Nb} \mathrm{K}$-edge EXAFS of PST-Ru and PSN-Ru, respectively
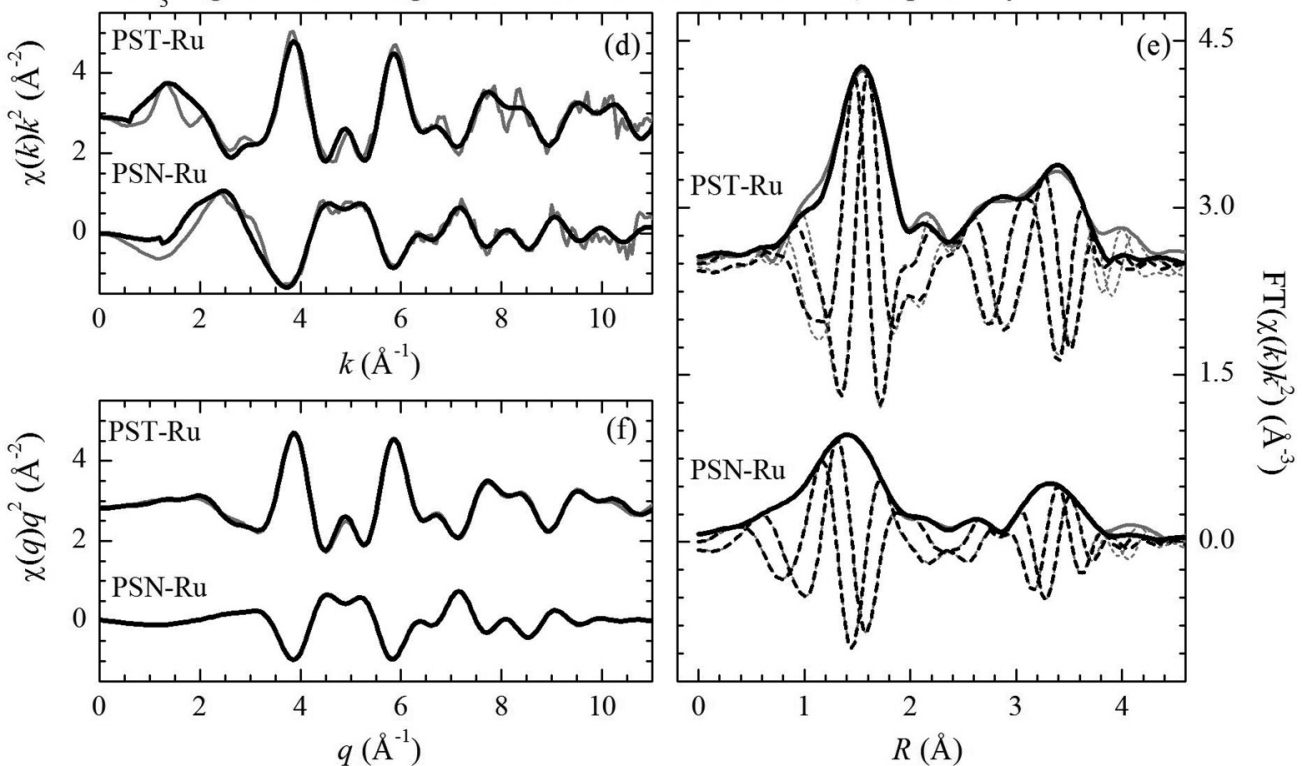

FIG. 6. Room-temperature EXAFS spectra at the Ru and $B^{\prime \prime}$-cation absorption edges of Ru-doped $\mathrm{PbSc}_{0.5} B^{\prime \prime}{ }_{0.5} \mathrm{O}_{3}, B^{\prime \prime}=\mathrm{Ta}$ or $\mathrm{Nb}$ : the weighted oscillatory parts $\chi(k) k^{3}$ for $\mathrm{Ru}(\mathrm{a})$ and $\chi(k) k^{2}$ for Ta/Nb (d), the FT in $R$ space (b) and (e), and the corresponding back FT $\chi(q) q^{3}$ (c) and $\chi(q) q^{2}$ (f). The spectra of PST-Ru are vertically off-shifted for clarity. Gray lines represent experimental data and bold black lines are the best fits; the dashed lines in (b) and (e) represent the real/imaginary parts of the Fourier transforms, while the solid lines represent the amplitudes. Note that in EXAFS the positions of the amplitude peaks in $R$ space appear at $R$ values smaller than the modeled atomic distances due to an uncorrected phase shift.

and $\mathrm{Ru}$ in PSN-Ru corresponds to the expected $N=6$, whereas in PST-Ru it is slightly below 6 for both Ta and Ru (Table III). A coordination number Nb-Sc $3.6 \pm 0.7$ slightly lower than the expected $N=6$ was also obtained for $\mathrm{Nb}$ $K$-edge PSN-Ru. A decrease in the coordination number may indicate the existence of incoherent distances, i.e., structural disorder in the corresponding coordination sphere. It should be noted, however, that deviations of the coordination number from the ideal value have to be considered with care because such deviations can be at least partially due to uncertainties in the amplitude reduction factor $S_{0}^{2}$ and the high correlation between $N$ and $S_{0}^{2}$ [34]. The uncertainties of $N$ given in Tables III and IV are entirely obtained from the fittings and do not include the influence of the uncertainty of $S_{0}^{2}$ on $N$. The expected value of $S_{0}^{2}$ was estimated by performing ab initio theoretical calculations using the code FEFF8.2. The calculated value of $S_{0}^{2}$ for $\mathrm{Ru}$, Ta, as well as for the $\mathrm{Nb}$ absorption edge was near 0.95 , and therefore $S_{0}^{2}$ was rounded and fixed to 1 during the fits. On the other hand, one can estimate the $S_{0}^{2}$ value for a certain experiment by measuring and evaluating the data of a reference material with known coordination. We have done this for $\mathrm{RuO}_{2}$ and obtained $S_{0}^{2}=0.8$, indicating 


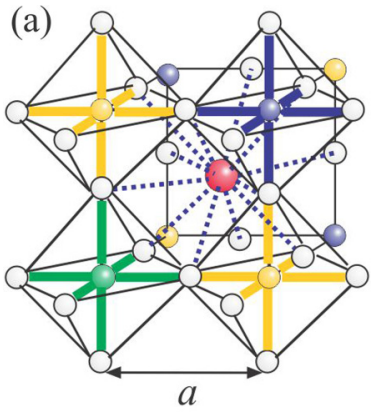

(b)
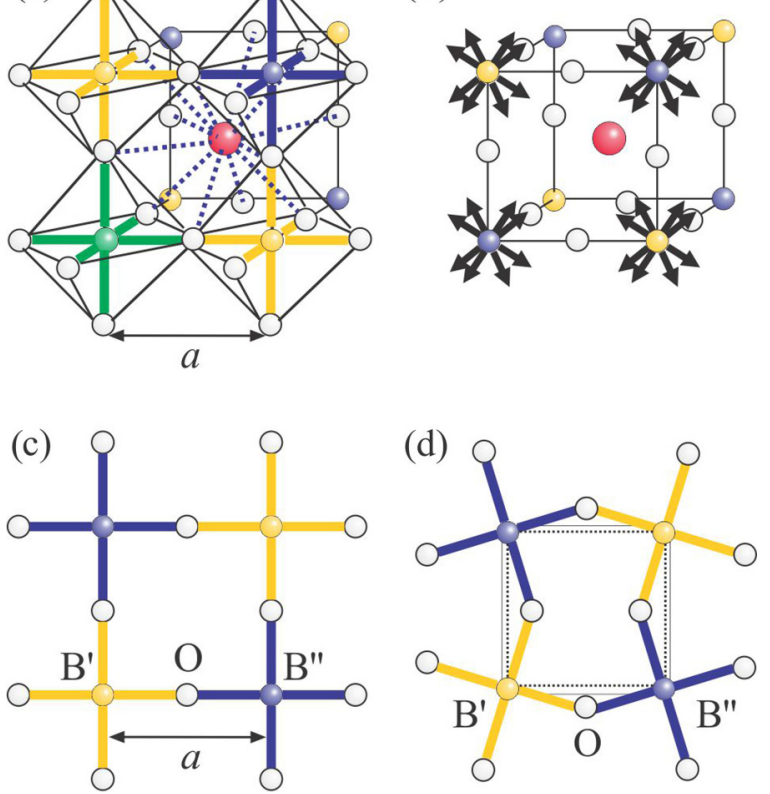

FIG. 7. (Color online) Sketch of chemical 1:1 B-site ordering in the vicinity of $\mathrm{Ru}$ incorporated into PST and PSN; red circle: $\mathrm{Pb}$; yellow: $\mathrm{Sc}$; green: $\mathrm{Ru}$; blue: $\mathrm{Ta} / \mathrm{Nb}$; white: $\mathrm{O}$. (a) Sketch of the eight-state order-disorder Potts model in which the $B$-site cations can randomly shift along one of the cubic $\langle 111\rangle$ directions. The arrows represent the off-centered displacements; for clarity the shifts of only half of the $B$-site cations are given (b). Projection of the $B \mathrm{O}_{6}$ system along the cubic [100] direction without (c) and with (d) octahedral tilts in the cubic (100) plane; the solid line in (d) represents the cubic unit cell and the dotted line represents the $B^{\prime}-B^{\prime \prime}$ distances.

that the $S_{0}^{2}$ value for Ru can be considered as $1 \pm 0.2$. Exact determination of the uncertainty in the $S_{0}^{2}$ value for Ta and $\mathrm{Nb}$ can hardly be performed without reference samples but it should be close to that for the uncertainty in the $S_{0}^{2}$ value for Ru. Since the product of $S_{0}^{2}$ and $N$ remains constant, if $S_{0}^{2}$ for Ta is smaller than $0.95-1.0$ predicted by $a b$ initio calculations, the coordination number $N_{\mathrm{Ta}-\mathrm{O}}$ should be larger, e.g., for $S_{0}^{2}=0.8 \mathrm{~N}$ is 5.4. Thus it would be speculative to assert that the lower coordination number is solid evidence for the existence of noncoherent distances, i.e., structural variations, but one can at least state that the trend in the coordination number for Ta-O suggests the existence of noncoherent distances. However, the value of $N_{\mathrm{Nb}-\mathrm{Sc}}=3.6 \pm 0.7$ in PSN-Ru does indicate the existence of incoherent distances.

Within the same compound, the oxygen coordination number of $\mathrm{Ru}$ is the same as that of the major $B^{\prime \prime}$ cation, i.e., EXAFS data do not provide any evidence for Jahn-Teller distortions of $\mathrm{RuO}_{6}$ octahedra suggested on the basis of Raman scattering but this can be due to the insufficient distance resolution $\Delta R \sim 0.09-0.10 \AA$ for the analyzed data sets, which is inversely proportional to the measured spectral range $\Delta k$ $(\Delta R \sim 1 / \Delta k)$.

The contribution of the $\mathrm{Pb}$ coordination sphere to the Ta $L_{3}$-edge EXAFS spectra could be best modeled assuming that $\mathrm{Pb}$ cations are off-centered with respect to the cubic $A$ site. The $\mathrm{Pb}$ coordination number for Ta in PST-Ru is $7.8 \pm 2.3$ at $3.47 \pm 0.02 \AA$ and it is close to the ideal cubic coordination number $N=8$. This indicates that $\mathrm{Pb}$ cations are either at the center of the $A \mathrm{O}_{12}$ cavity or they are coherently off-centered in pairs and the correlated $\mathrm{Pb}$ displacements are antiphase to ensure constructive interference of the scattered photoelectron, i.e., in a certain extent there is local antipolar order of $\mathrm{Pb}$ atoms. A large number of structural studies of $\mathrm{Pb}$-based relaxors including pair-distribution function analysis [48,49], nuclear magnetic resonance spectroscopy [50], and Raman spectroscopy $[23,41,46]$ indicate that $\mathrm{Pb}$ atoms are offcentered. Hence the Ta $L_{3}$-edge EXAFS data presented here suggest a predominance of correlated antiphase displacements of $\mathrm{Pb}$ in the PST host matrix. The possible combinations of $\mathrm{Pb}$ displacements are, however, too many to claim a predominant direction of $\mathrm{Pb}$ off-centering and a mean value of the $\mathrm{Pb}$ shifts. The contribution of the $\mathrm{Pb}$ coordination sphere to the $\mathrm{Ru}$ and Nb $K$-edge EXAFS spectra could also be best modeled with off-centered $\mathrm{Pb}$ cations. The $\mathrm{Pb}$ coordination number of $\mathrm{Nb}$ in PSN-Ru is close to zero. Therefore, one can conclude that the off-center displacements of $\mathrm{Pb}$ in the PSN host matrix are rather incoherent. Off-centered $\mathrm{Pb}$ displacements that on the local scale are much more strongly correlated in pure PST than in pure PSN have been also deduced from neutron diffraction data [24]. The intensity ratio of the $\bar{Z}(X Y) Z$ Raman scattering near 350 and $300 \mathrm{~cm}^{-1}$ correlates with the length of coherence of ferroic $\mathrm{Pb}-\mathrm{O}$ distortions within planes perpendicular to the cubic body diagonal [41]. This ratio measured with the same wavelength $514.5 \mathrm{~nm}$ is $0.19 \pm 0.02$ and $0.08 \pm 0.03$ for PST-Ru and PSN-Ru, respectively, and it is hence in good agreement with the degree of positional disorder of $\mathrm{Pb}$ deduced from EXAFS data. The $\mathrm{Pb}$ coordination number for $\mathrm{Ru}$ suggests that the $\mathrm{Ru}-\mathrm{Pb}$ distances in both PST-Ru and PSN-Ru exhibit a low degree of coherence as compared to the $\mathrm{Ta}-\mathrm{Pb}$ distances but they are somehow better correlated than the $\mathrm{Nb}-\mathrm{Pb}$ distances.

The distances $\mathrm{Ta}-\mathrm{Sc}, \mathrm{Nb}-\mathrm{Sc}$, and $\mathrm{Ru}-\mathrm{Sc}$ derived from the EXAFS spectra are shorter than the expected cubic $B^{\prime}$ $B^{\prime \prime}$ distances calculated from the cubic unit-cell parameter determined by XRD. This cannot be ascribed to the $B$-site cation hopping between the eight possible orientation states [see Fig. 7(b)] because, as pointed out above, the statistically calculated mean $B^{\prime}-B^{\prime \prime}$ distance is even slightly longer than the cubic unit-cell parameter. The only situation in which the $B^{\prime}$ $B^{\prime \prime}$ distances are shorter than the cubic cell parameter is when octahedral tilting exists [see Figs. 7(c) and 7(d)]. Assuming local octahedral tilts and using simple geometrical relations, we have calculated the Sc-O-Ta, Sc-O-Nb, and Sc-O-Ru bond angles from the atomic distances (see Table III). The obtained values considerably deviate from $180^{\circ}$ but $B-\mathrm{O}-B$ angles of similar magnitude have been observed in perovskites with a small tolerance factor $t=\frac{r_{\text {ionic }}(A)-r_{\text {ionic }}(O)}{\sqrt{2}\left[r_{\text {ionic }}(B)-r_{\text {ionic }}(O)\right]}$ and long-range ferroelectric order such as $\mathrm{LiNbO}_{3}$ and $\mathrm{BiFeO}_{3}$ [51,52]. Still it is puzzling that according to XRD the structure is cubic, i.e., no tilting, whereas the EXAFS data suggest local octahedral tilting $\varphi=\left[180-\angle\left(B^{\prime}-\mathrm{O}-B^{\prime \prime}\right)\right] / 2$ of approximately $20^{\circ}$ not only in the vicinity of Ru dopant but also around the major $B^{\prime \prime}$ cation. We attribute this discrepancy to the difference in the length scale as well as in the time scale of sensitivity of the two methods $[53,54]$. If the structure of the studied relaxors were simply cubic and homogeneous, without any dynamical ferroic atomic arrangements, then the $B^{\prime}-B^{\prime \prime}$ distances measured by 
EXAFS may be slightly longer but not shorter than the $B^{\prime}-B^{\prime \prime}$ distances derived from the unit-cell parameters, due to the different effect of the thermal vibrations on the average distance between atoms (EXAFS) and the average distance between the lattice points (XRD) [55]. However, it is well known that the relaxor structure is highly inhomogeneous and near room temperature it is comprised of dynamic ferroic nanoregions of coupled ferroic distortions with a lifetime $\sim 10^{-5} \mathrm{~s}$ [50] and a size $\sim 3 \mathrm{~nm}[56]$ as well as of uncoupled local ferroic distortions with much faster hopping dynamics. Depending on the time and length scale of sensitivity of the probe method, one can observe "more" or "less" abundant ferroic fraction: the fraction of coupled ferroic distortions. For XRD it is none or negligibly small because the Bragg diffraction peaks represent the time average of ferroic nanoregions in all possible orientation states, which is a cubic structure with a unit-cell parameter equal to the mean $B$ - $B$ distance averaged over different ferroic nanoregions. The correlated ferroic distortions within a separate ferroic nanoregion appear in the single-crystal XRD pattern as X-ray diffuse scattering streaks (see Fig. 1), while the uncorrelated ferroic distortions most probably disappear in the background. On the other hand, due to its time- and length-scale sensitivity [53,54] EXAFS can detect correlated local dynamic structural distortions in a sphere $\sim 6 \AA$ around the absorbing atoms which have a lifetime longer than $10^{-15} \mathrm{~s}$. Therefore, EXAFS can make a "snapshot" of the structure within a separate ferroic nanoregion existing at a given time, while the uncoupled ferroic distortions result mainly in a reduction of the coordination number and/or go into the background. Therefore, we suggest that the shorter $B^{\prime}-B^{\prime \prime}$ distances detected by EXAFS are due to dynamic intermediate-range octahedral tilting, existing along with the cation off-centered displacements. This result is in full accordance with recent DFT calculations of chemically $B$-site ordered PST and PSN demonstrating that the most energetically favored structural state at low pressures is composed of polar cation shifts and antiphase octahedra [24]. The existence of mesoscopic-scale chemical $B$-site order has been established by several experimental methods $[19,23,56,57]$ and hence, mesoscopic-scale octahedral tilting along this cation off-centered displacement should be expected for relaxors. Furthermore, static long-range order of octahedral tilts in relaxors has been observed at low temperatures [40] as well as at high pressures [5,24,58], which most probably is developed from the dynamic intermediate-range tilt order existing at room temperature and ambient pressure revealed by the EXAFS data presented here. The pressure evolution of the phonon anomalies observed by Raman spectroscopy, which has a time-scale sensitivity of $\sim 10^{-12} \mathrm{~s}$, also indicates the existence of intermediate-range antiferrodistortive order involving octahedral tilts already at ambient conditions [5,58]. The existence of antiphase octahedral tilts above and below the Curie temperature was also demonstrated in $\mathrm{PbZr}_{1-x} \mathrm{Ti}_{x} \mathrm{O}_{3}$ by high-resolution neutron diffraction [59]. Hence, the coexisting cation off-centering and octahedral tilting may play a critical role for the properties and performance of perovskite-type ferroelectrics.

It should be mentioned that according to the atomic distances derived from EXAFS data published by Frenkel et al [19], the Sc-O-Ta angle in pure PST is $165^{\circ}$, which gives a dynamic tilt angle of $7.5^{\circ}$. Similar values of static long-range ordered tilting were detected in pure PST at low temperature and ambient pressure [40], in PST and PSN at high pressures and room temperature, as well as in PSN heavily doped with La at ambient conditions [45].

The cubic unit-cell parameters of PST-Ru and PSN-Ru determined by XRD (Table I) are slightly smaller than the corresponding unit-cell parameters of pure PST [19,41] and PSN [42], which is consistent with the larger value of dynamic octahedral tilting in Ru-doped PST as compared to that of PST. The tilting around Ru in both PST-Ru and PSN-Ru as well as around $\mathrm{Nb}$ in the PSN matrix is smaller than the tilting around Ta in the PST matrix. This correlates with the higher incoherence in the $\mathrm{Ru}-\mathrm{Pb}$ and $\mathrm{Nb}-\mathrm{Pb}$ distances as compared to

Ru K-edge EXAFS of PZN-0.1PT-Ru
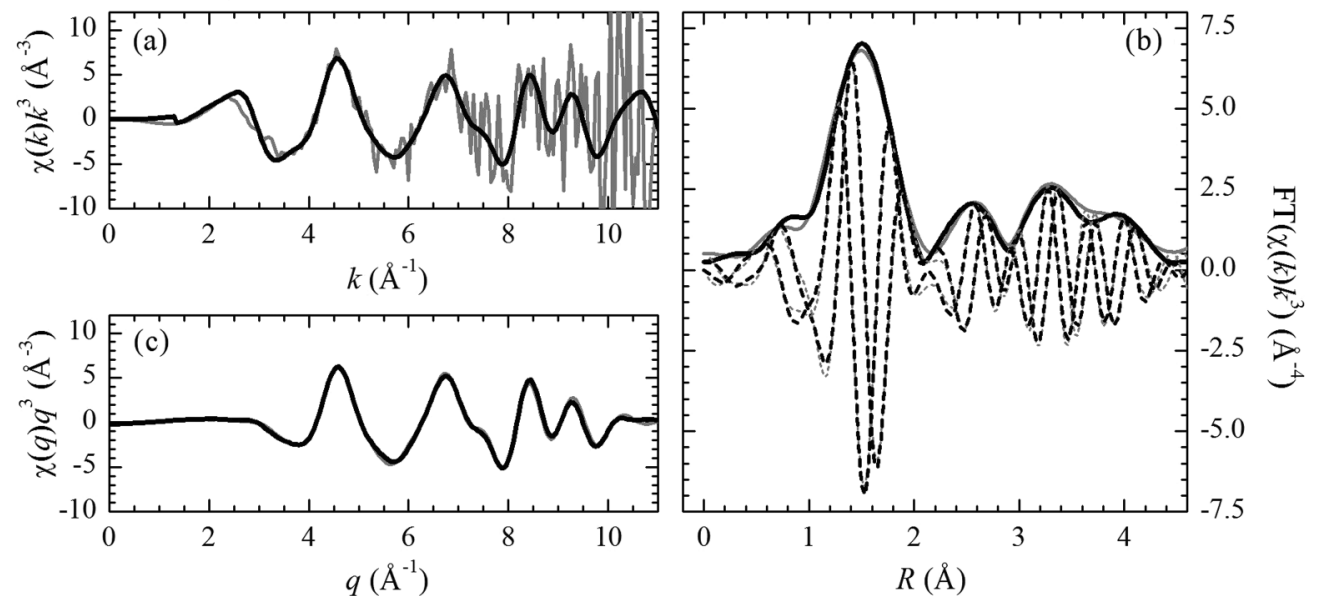

FIG. 8. Room-temperature EXAFS spectra at the Ru $K$ edge of PZN-0.1PT-Ru: the weighted oscillatory part $\chi(k) k^{3}$ (a), its FT in $R$ space (b), and the corresponding back FT $\chi(q) q^{3}$ (c). Gray lines represent experimental data and bold black lines are the best fits; the dashed lines in (b) represent the real/imaginary parts of the FT, while the solid lines represent the amplitudes. Note that in EXAFS, the positions of the amplitude peaks in $R$ space appear at $R$ values smaller than the modeled atomic distances due to an uncorrected phase shift. 
$\mathrm{Ta}-\mathrm{Pb}$, suggesting that dynamic octahedral tilting is supported by correlated antiphase off-center displacements of $\mathrm{Pb}$, which in the relaxor ergodic state are also of dynamic nature.

\section{PZN-0.1PT-Ru}

The Ru $K$-edge EXAFS spectra of PZN-0.1PT-Ru are shown in Fig. 8, while the atomic distances obtained from the best-fit model are given in Table IV. One can clearly see that the way of incorporation of $\mathrm{Ru}$ into the PZN-0.1PT matrix considerably differs from the way $\mathrm{Ru}$ enters the structure of PST and PSN. Similar to PST-Ru and PSN-Ru, the Ru cations are octahedrally coordinated to the nearest oxygen anions but in contrast to PST-Ru and PSN-Ru, the mean $\mathrm{Ru}-\mathrm{O}$ distance in PZN-0.1PT-Ru is equal to half of the pseudocubic unit-cell parameter, i.e., oxygen atoms are not shifted towards $\mathrm{Ru}$. There are two types of ferroelectrically active cations in the PZN-0.1PT host matrix: $\mathrm{Nb}^{5+}$ and $\mathrm{Ti}^{4+}$ and similarly to PST-Ru and PSN-Ru, there are no ferroelectrically active $B$-site cations in the surroundings of $\mathrm{Ru}$ embedded into PZN-0.1PT. This suggests that $\mathrm{Ru}$ may substitute for $\mathrm{Nb}$ and/or Ti. However, in the surroundings of $\mathrm{Ru}$ there is $\mathrm{Zn}$ as well as $\mathrm{Ru}$ and the $\mathrm{Ru}-\mathrm{Zn}$ and $\mathrm{Ru}-\mathrm{Ru}$ are considerably smaller than the cubic $B-B$ distance. In fact the $\mathrm{Ru}-\mathrm{Ru}$ distance matches well the cubic distance between the $B$ and $A$ sites. Furthermore, the $\mathrm{Pb}$ coordination number of $\mathrm{Ru}$ is approximately 6 and the average $\mathrm{Ru}-\mathrm{Pb}$ distance is very close to the $A-A$ distance, indicating that there is $\mathrm{Ru}$ on the $A$ site. Therefore we suggest that in PZN-0.1PT Ru shows affinity to cluster, namely, to form $\mathrm{RuO}_{6}$ chainlike structural species along the cubic body diagonal (see Fig. 9). The $\mathrm{RuO}_{6}$ octahedra should be elongated along the local threefold axis of symmetry in order to adopt $A-\mathrm{O}$ bonds close to $B-\mathrm{O}$ bonds. The $\mathrm{ZnO}_{6}$ octhadera in the vicinity of $\mathrm{Ru}$ should also be rather irregular to maintain the bond connectivity. The relatively short $\mathrm{Ru}-\mathrm{Zn}$ distance along with the small coordination number

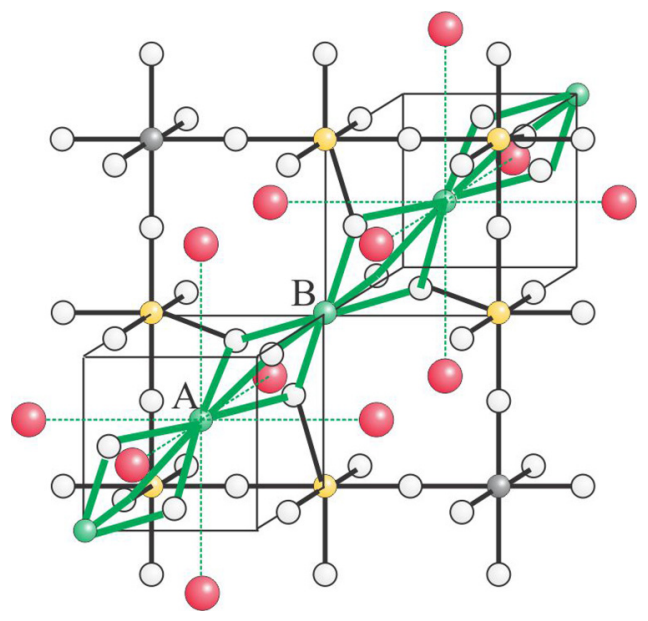

FIG. 9. (Color online) Sketch of possible clustering of Ru incorporated into PZN-0.1PT; red circles: $\mathrm{Pb}$; yellow: $\mathrm{Zn}$; green: $\mathrm{Ru}$; gray: $\mathrm{Nb} / \mathrm{Ti} / \mathrm{Zn}$; white: $\mathrm{O}$. For simplicity the $\mathrm{Zn}$ atoms are placed in the $B$ site of the aristotype $A B \mathrm{O}_{3}$ structure, although EXAFS data of $\mathrm{PZN}-0.1-\mathrm{PT}-\mathrm{Ru}$ indicate positional disorder of $\mathrm{Zn}$ in the vicinity of Ru.
(Table IV) indicate that $\mathrm{Zn}$ cations are most probably shifted towards $A$-positioned $\mathrm{Ru}$ and exhibit substantial positional disorder.

\section{CONCLUSIONS}

The incorporation of $\mathrm{Ru}$ in both $\mathrm{Pb} B^{\prime}{ }_{0.5} \mathrm{~B}^{\prime \prime}{ }_{0.5} \mathrm{O}_{3}$ and $(1-x) \mathrm{Pb}^{\prime}{ }_{1 / 3} B^{\prime \prime}{ }_{13} \mathrm{O}_{3}-x \mathrm{PbTiO}_{3}$ relaxor types interferes with the development of long-range ferroelectric order upon temperature decrease and shifts the room-temperature optical absorption edge towards lower energies. The spectroscopic analyses of PST-Ru, PSN-Ru, and PZN-0.1PT-Ru, however, reveal that $\mathrm{Ru}$ is embedded in $\mathrm{Pb} B^{\prime}{ }_{0.5} B^{\prime \prime}{ }_{0.5} \mathrm{O}_{3}$ in a manner different from that for $(1-x) \mathrm{Pb}^{\prime}{ }_{1 / 3} B^{\prime \prime}{ }_{2 / 3} \mathrm{O}_{3}-x \mathrm{PbTiO}_{3}$ near MPB. In both relaxor matrices $\mathrm{Ru}$ is octahedrally coordinated but the average oxidation state of $\mathrm{Ru}^{n+}$ in $\mathrm{Pb} B^{\prime}{ }_{0.5} B^{\prime \prime}{ }_{0.5} \mathrm{O}_{3}$ is $\sim 4.4$, whereas $n \sim 3.8$ in $\left(1-x_{\mathrm{MPB}}\right) \mathrm{Pb} B^{\prime}{ }_{1 / 3}$ $B^{\prime \prime}{ }_{2 / 3} \mathrm{O}_{3}-x_{\mathrm{MPB}} \mathrm{PbTiO}_{3}$.

According to EXAFS data on PST-Ru and PSN-Ru, Ru is homogeneously dispersed as a point substitution defect in $\mathrm{Pb} B^{\prime}{ }_{0.5} B^{\prime \prime}{ }_{0.5} \mathrm{O}_{3}$ and it exclusively replaces the ferroelectrically active $B^{\prime \prime}$ cation. Chemical 1:1 $B$-site order is observed around $\mathrm{Ru}$ as well as around the major $B^{\prime \prime}$ cation, independently of the low degree or absence of long-range chemical order. Furthermore, dynamic $\mathrm{BO}_{6}$ tilting is observed in the vicinity of both the $\mathrm{Ru}$ dopant and the major $B^{\prime \prime}$ cation, regardless of the fact that the average structure appears cubic in XRD experiments at ambient conditions. Lead cations in the surroundings of the $B^{\prime \prime}$ cations as well as of $\mathrm{Ru}$ are off-center displaced from the $A$ site in the aristotype cubic $A B \mathrm{O}_{3}$. The local $\mathrm{Pb}$-position order in terms of coherent $B-\mathrm{Pb}$ distances is high for $\mathrm{Ta}$, considerably decreases near $\mathrm{Ru}$, and almost absent near $\mathrm{Nb}$. Raman scattering suggests electron-phonon coupling and Jahn-Teller distortions in the vicinity of $\mathrm{Ru}$ in $\mathrm{Pb} B^{\prime}{ }_{0.5} B^{\prime \prime}{ }_{0.5} \mathrm{O}_{3}$, which might be responsible for the observed large redshift of the optical absorption edge.

According to EXAFS data on PZN-0.1PT-Ru, Ru incorporated into $\left(1-x_{\mathrm{MPB}}\right) \mathrm{Pb} B^{\prime}{ }_{1 / 3} B^{\prime \prime}{ }_{2 / 3} \mathrm{O}_{3}-x_{\mathrm{MPB}} \mathrm{PbTiO}_{3}$ has the affinity to cluster, by substituting both $A$ and $B$ cations and forming chainlike structural species of face-sharing $\mathrm{RuO}_{6}$ octahedra elongated along one of the threefold axes of symmetry.

The comparison of the $\mathrm{Ru} K$-edge EXAFS results for the three compounds indicate that $A B^{\prime}{ }_{1 / 2} B^{\prime \prime}{ }_{1 / 2} \mathrm{O}_{3}$ matrices favor the incorporation of $\mathrm{Ru}$ as point substitution defect exclusively on the $B$ site and this seems to be the reason for the local Jahn-Teller effect suggested by multiwavelength Raman spectroscopy and the huge shift of the optical absorption edge.

\section{ACKNOWLEDGMENTS}

Financial support by the Deutsche Forschungsgemeinschaft (MI 1127/5-1) is gratefully acknowledged. The authors thank Jianmin Shi, Technische Universität Braunschweig, for measuring the optical spectra of pure and Ru-doped PZN-0.1PT and Bernd Maier, Ludwig-Maximilians Universität München, for help with XRD data. We acknowledge the Synchrotron Light Source ANKA for provision of instruments at their beamlines. 
[1] A. A. Bokov and Z.-G. Ye, J. Mater. Sci. 41, 31 (2006).

[2] G. A. Samara, J. Phys.: Condens. Matter 15, R367 (2003).

[3] R. A. Cowley, S. N. Gvasaliya, S. G. Lushnikov, B. Roessli, and G. M. Rotaru, Adv. Phys. 60, 229 (2010).

[4] L. E. Cross, in Piezoelectricity. Evolution and Future of a Technology, edited by W. Heywang, K. Lubitz, W. Wersing, Springer Series in Materials Science (Springer-Verlag, Berlin, 2008), Vol. 114, p. 131

[5] B. Mihailova, N. Waeselmann, B. J. Maier, A.-M. Welsch, R. J. Angel, and U. Bismayer, High Pressure Res. 33, 595 (2013).

[6] J. F. Scott, ISRN Mater. Sci. 2013, 187313 (2013).

[7] O. Kircher and R. Böhmer, Eur. Phys. J. B 26, 329 (2002).

[8] S. E. Park and T. R. Shrout, J. Appl. Phys. 82, 1804 (1997).

[9] C. H. Lin, C. Y. Huang, and J. Y. Chang, Appl. Surf. Sci. 208-209, 340 (2003).

[10] K. Buse, H. Hesse, U. van Stevendaal, S. Loheide, D. Sabbert, and E. Kratzig, Appl. Phys. A 59, 563 (1994).

[11] P. Clem and D. Payne, J. Appl. Phys. 77, 5865 (1995).

[12] T. Kida, R. Kammuri, M. Hagiwara, S. Yoshii, W. Kobayashi, M. Iwakawa, and I. Terasaki, Phys. Rev. B 85, 195122 (2012).

[13] T. L. Phan, Y. D. Zhang, S. C. Yu, P. Q. Thanh, and P. D. H. Yen, J. Korean Phys. Soc. 61, 1568 (2012).

[14] P. A. Kumar, R. Mathieu, R. Vijayaraghavan, S. Majumdar, O. Karis, P. Nordblad, B. Sanyal, O. Eriksson, and D. D. Sarma Phys. Rev. B 86, 094421 (2012).

[15] T. Scholz, B. Mihailova, G. A. Schneider, T. Malcherek, V. Marinova, M. Gospodinov, N. Pagels, J. Heck, and U. Bismayer, J. Appl. Phys. 106, 074108 (2009).

[16] T. Vitova, J. Hormes, K. Pethmann, and T. Woike, Phys. Rev. B 77, 144103 (2008).

[17] T. Vitova, J. Hormes, M. Falk, and K. Buse, J. Appl. Phys. 105, 013524 (2009).

[18] A. Mesquita, A. Michalowicz, and V. R. Mastelaro, J. Appl. Phys. 111, 104110 (2012).

[19] A. I. Frenkel, D. M. Pease, J. Giniewicz, E. A. Stern, D. L. Brewe, M. Daniel, and J. Budnick, Phys. Rev. B 70, 014106 (2004).

[20] I. W. Chen, P. Li, and Y. Wang, J. Phys. Chem. Solids 57, 1525 (1996).

[21] I. W. Chen, J. Phys. Chem. Solids 61, 197 (2000).

[22] T. Egami, Ferroelectrics 267, 101 (2002).

[23] N. Waeselmann, B. Mihailova, B. J. Maier, C. Paulmann, M. Gospodinov, V. Marinova, and Bismayer, Phys. Rev. B 83, 214104 (2011).

[24] B. J. Maier, N. Waeselmann, B. Mihailova, R. J. Angel, C. Ederer, C. Paulmann, M. Gospodinov, A. Friedrich, and U. Bismayer, Phys. Rev. B 84, 174104 (2011).

[25] E. Dul'kin, B. Mihailova, M. Gospodinov, and M. Roth, Eur. Phys. Lett. 94, 57002 (2011).

[26] E. Dul'kin, B. Mihailova, M. Gospodinov, and M. Roth, J. Appl. Phys. 113, 054105 (2013).

[27] B. J. Maier, T. Steilmann, M. Gospodinov, U. Bismayer, and B. Mihailova, J. Appl. Phys. 112, 124111 (2012).

[28] T. Steilmann, B. J. Maier, M. Gospodinov, U. Bismayer, and B. Mihailova, J. Phys.: Condens. Matter 26, 175401 (2014).

[29] C. G. F. Stenger and A. J. Burggaaf, Phys. Status Solidi A 61, 275 (1980).

[30] B. Noheda, D. E. Cox, G. Shirane, S. E. Park, L. E. Cross, and Z. Zhong, Phys. Rev. Lett. 86, 3891 (2001).

[31] G. Xu, J. Phys. Soc. Jpn. 79, 011011 (2010).
[32] N. Waeselmann, B. Mihailova, B. J. Maier, R. J. Angel, J. Zhao, C. Paulmann, M. Gospodinov, N. Ross, and U. Bismayer, Phys. Rev B 85, 014106 (2012).

[33] B. Ravel and M. Newville, J. Synchrotron Radiat. 12, 537 (2005).

[34] J. J. Rehr and R. C. Albers, Rev. Mod. Phys. 72, 621 (2000).

[35] See Supplemental Material at http://link.aps.org/supplemental/ 10.1103/PhysRevB.89.144112 for a detailed description of the strategy in evaluating EXAFS spectra and the considered scattering-path models, focusing on Ta L3-edge EXAFS data of PST-Ru.

[36] G. Dalba, P. Fornasini, R. Gotter, and F. Rocca, Phys. Rev. B 52, 149 (1995).

[37] A. S. Chaves, F. C. S. Barreto, R. A. Nogueira, and B. Zeks, Phys. Rev. B 13, 207 (1976).

[38] M. Paściak and R. Welberry, Z. Kristallogr. 226, 113 (2011).

[39] A. Bosak, D. Chernyshov, S. Vakhrushev, and M. Krisch, Acta Crystallogr., Sect. A: Found. Crystallogr. 68, 117 (2012).

[40] P. M. Woodward and K. Z. Baba-Kishi, J. Appl. Crystallogr. 35, 233 (2002).

[41] B. Mihailova, B. J. Maier, C. Paulmann, T. Malcherek, J. Ihringer, M. Gospodinov, R. Stosch, B. Güttler, and U. Bismayer, Phys. Rev. B 77, 174106 (2008).

[42] B. J. Maier, B. Mihailova, C. Paulmann, J. Ihringer, M. Gospodinov, R. Stosch, B. Güttler, and U. Bismayer, Phys. Rev. B 79, 224108 (2009).

[43] A.-M. Welsch, B. J. Maier, B. Mihailova, R. J. Angel, J. Zhao, C. Paulmann, J. M. Engel, M. Gospodinov, V. Marinova, and U. Bismayer, Z. Kristallogr. 226, 126 (2011).

[44] Detailed analysis of the resonance Raman scattering of perovskite-type relaxors is beyond the scope of this paper and will be given in G. de la Flor Martin, M. Wehber, A. Rohrbeck, M. Aroyo, U. Bismayer, and B. Mihailova (unpublished).

[45] B. J. Maier, A.-M. Welsch, B. Mihailova, R. J. Angel, J. Zhao, C. Paulmann, J. M. Engel, W. G. Marshall, M. Gospodinov, D. Petrova, and U. Bismayer, Phys. Rev. B 83, 134106 (2011).

[46] J. Toulouse, F. Jiang, O. Svitelskiy, W. Chen, and Z.-G. Ye, Phys. Rev. B 72, 184106 (2005).

[47] A. Slodczyk, P. Daniel, and A. Kania, Phys. Rev. B 77, 184114 (2008).

[48] T. Egami, Annu. Rev. Mater. Res. 37, 297 (2007).

[49] I.-K. Jeong, J. K. Lee, and R. H. Heffner, Appl. Phys. Lett. 92, 172911 (2008).

[50] R. Blinc, A. Gregorovic, B. Zalar, R. Pirc, V. V. Laguta, and M. D. Glinchuk, Phys. Rev. B 63, 024104 (2000).

[51] S. C. Abrahams, W. C. Hamilton, and J. M. Reddy, J. Phys. Chem. Solids 27, 1013 (1966).

[52] I. Sosnowska, W. Schaefer, W. Kockelmann, and I. O. Troyanchuk, Mater. Sci. Forum 378, 616 (2001).

[53] J. Stöhr, in NEXAFS Spectroscopy, Springer Series in Surface Sciences (Springer-Verlag, Berlin, 2003), Vol. 25.

[54] The XRD technique is based on elastic scattering from periodic atomic arrays and the diffraction peaks are formed from the constructive interference of the electromagnetic waves reflected from periodic atomic planes, i.e., in order to observe diffraction peaks from a solid there should be repetitions of the unit cell of the order of 10 , which is commonly considered an established long-range order. Atomic formations on shorter 
length scales, i.e., intermediate-range order and short-range order [following the classification in J. M. Ziman, Models of Disorder (Cambridge University Press, Cambridge, UK, 1976)] generate the so-called $\mathrm{x}$-ray diffuse scattering (observed also from the materials studied here; see Fig. 1) and/or contribute to the background of the x-ray diffraction pattern. Furthermore, the Bragg peak positions represent the interplanar spacings formed by the equilibrium atomic positions, whereas any dynamical structural fluctuations including crystal phonon modes and short-lifetime ferroic distortions hopping between different orientation states are averaged in the atomic displacement ellipsoids. In short, the diffraction peak positions and the consequently obtained unit-cell parameters are essentially time averaged. In strong contrast, EXAFS is based on the absorption of $\mathrm{x}$ rays by core electrons and the subsequent scattering of the excited electrons from the surrounding atoms, i.e., the interaction events are very fast, of the order of $10^{-15} \mathrm{~s}$, and localized around the absorbing atom within about $6 \AA$. This means that EXAFS is a technique very sensitive to the shortand intermediate-range order and capable of detecting correlated local dynamic structural distortions with a lifetime longer than $10^{-15} \mathrm{~s}$.

[55] P. Fornasini, S. a Beccara, G. Dalba, R. Grisenti, A. Sanson, M. Vaccari, and F. Rocca, Phys. Rev. B 70, 174301 (2004).

[56] L. A. Bursill, P. Julin, Q. Hua, and N. Setter, Phys. B (Amsterdam, Neth.) 205, 305 (1995).

[57] Y. Yan, S. J. Pennycook, Z. Xu, and D. Viehland, Appl. Phys. Lett. 72, 3145 (1998).

[58] B. Mihailova, R. J. Angel, B. J. Maier, A.-M. Welsch, J. Zhao, M. Gospodinov, and U. Bismayer, IEEE T. Ultrason. Ferr. 58 , 1905 (2011).

[59] N. Zhang, H. Yokota, A. M. Glazer, and P. A. Thomas, Acta Crystallogr., Sect. B: Struct. Sci. 67, 461 (2011). 